\title{
(Mis-)handling gauge invariance in the theory of the quantum Hall effect II: Perturbative results
}

\author{
M.A. Baranov*, A.M.M. Pruisken, B. Škorić \\ Institute for Theoretical Physics, University of Amsterdam, Valckenierstraat 65, \\ 1018 XE Amsterdam, The Netherlands
}

(October 9, 1998)

\begin{abstract}
The concept of $\mathcal{F}$-invariance, which previously arose in our analysis of the integral and half-integral quantum Hall effects, is studied in $2+2 \varepsilon$ spatial dimensions.

We report the results of a detailed renormalization group analysis and establish the renormalizability of the (Finkelstein) action to two loop order. We show that the infrared behaviour of the theory can be extracted from gauge invariant ( $\mathcal{F}$-invariant) quantities only. For these quantities (conductivity, specific heat) we derive explicit scaling functions.

We identify a bosonic quasiparticle density of states which develops a Coulomb gap as one approaches the metal-insulator transition from the metallic side. We discuss the consequences of $\mathcal{F}$-invariance for the strong coupling, insulating regime.
\end{abstract}

PACSnumbers 72.10.-d, 73.20.Dx, 73.40.Hm

Typeset using REVTEX 


\section{INTRODUCTION}

\section{A. General introduction}

Two of the authors recently proposed a topological extension of the Finkelstein nonlinear sigma model for localization and interaction effects $t$. This theory was motivated by the extended experimental work on scaling in the quantum Hall regime by H.P. Wei et

al. 2 . It was shown that the interacting electron gas shares many of the features which were previously found for free electrons in a magnetic field. In particular, the same scaling diagram was obtained, indicating that the topological concept of instanton vacuum, rather than being a free particle theory of the plateau transitions alone, presumably has a much more profound significance in the theory of quantum transport.

In a previous papen we elaborated on the microscopic origins of the effective sigma model action. We recognized a new symmetry in the problem (' $\mathcal{F}$-invariance') which previously has gone largely unnoticed and which is intimately related to the electrodynamic $U(1)$ gauge invariance of the theory.

For ordinary metallic conductors the consequences of $\mathcal{F}$-invariance may be summarized by saying that the Einstein relation between conduction and diffusion no longer describes the process of quantum transport. Instead, the internally generated electric field due to the Coulomb interactions is what enters into the transport equations. We have shown that this aspect of ordinary metals has direct consequences for the composite fermion approach to the half-integral quantum Hall effect.

The half-integral effect, along with the transport problem in weak magnetic fields, is described by our topological action by working with the Chern-Simons or external fields in weak coupling or tree level approximation. On the other hand, in subsequent papers 6 we will address the Luttinger liquid theory of the chiral fractional quantum Hall edge statest. The Luttinger liquid can be microscopically obtained from our topological action by working in the opposite limit of strong coupling.

The Fermi-liquid state of the half-integral effect and the Luttinger liquid state of the edges are completely different physical scenarios which are actively and separately being pursued in the recent literature on the quantum Hall effect. Since they appear as the extreme weak and strong coupling limits of a single topological action, it becomes possible to pursue a more ambitious program and see how they are related.

In this paper we report the results of detailed renormalization group studies of the perturbative weak coupling regime. Our main objective is to further investigate the fundamental consequences of $\mathcal{F}$-invariance and to establish the renormalizability of the theory to two loop order. In section [I] we present a different version of Finkelstein's momentum shell computations 8 , namely the background field method in dimensional regularization, which leads to major computational advantages. One of the most important consequences of the renormalization group procedure, however, is that the infrared behaviour of the theory can be extracted from $\mathcal{F}$-invariant quantities or correlation functions only. This aspect of the problem has till now remained completely unnoticed.

For arbitrary correlation functions or renormalization group procedures which are not $\mathcal{F}$ invariant, the perturbative expansions are generally plagued by infrared problems which can 
not be resolved by the renormalization group. This means, for example, that the expansion procedure can not be used to show that the diffusion propagator (which is not an $\mathcal{F}$-invariant quantity) contains an infrared cutoff of the type $1 / \tau_{\text {in }}$, i.e. the inelastic scattering length as naively obtained from the Golden Rule. For the same reason it is also fundamentally incorrect to interpret the theory in terms of a 'Fermi-liquid with length scale dependent parameters 1 .

Examples of $\mathcal{F}$-invariant quantities are the linear response formulae which involve current and density correlation functions and, of course, the free energy or grand canonical potential itself. The most important results of this work are reported in Sections $\mathbb{I I}$ and $\mathbb{\Pi \nabla}$ where we compute these quantities and in Section $\mathrm{V}$ where we derive explicit scaling functions for them.

Based on a two-loop expansion for the free energy (Section IVC and the Appendix) we are able to identify a new quantity in the problem, namely a bosonic quasiparticle density of states which enters the expression for the specific heat (Section $\nabla \mathbb{1}$ ). This quantity develops a Coulomb gap as one approaches the metal-insulator transition in $2+2 \varepsilon$ dimensions from the metallic side (Section VIB).

As a logical follow-up we next discuss the problem of the strong coupling, insulating phase (Section VID). We show that important progress can be made by relying on the more familiar but completely analogous theory of the classical Heisenberg ferromagnet. Our results indicate that the insulating phase is dominated by additional terms in the action which are usually dismissed as being 'irrelevant'. This, then, will serve as a starting point for an extended renormalization group program. Progress along these lines, along with the consequences of the Coulomb interactions for the plateau transitions in the quantum Hall regime, will be reported elsewhere.

\section{B. Preliminaries}

\section{Formalism}

In this and the following section we summarize the main results of the $Q$-field theory for the quantum Hall effect as discussed in great detail in our previous work 5 . The electronic degrees of freedom in this theory are contained in a matrix field $Q$, for which the following effective action was derived,

$$
\begin{aligned}
Z[A]= & \int \mathcal{D} Q e^{S[Q, A]} ; \quad S[Q, A]=S_{\sigma}[Q, A]+S_{\mathrm{F}}[Q]+S_{\mathrm{U}}[Q, A] \\
S_{\sigma}[Q, A]= & -\frac{1}{8} \sigma_{x x}^{0} \operatorname{Tr}\left[\partial_{j}-i \hat{A}_{j}, Q\right]\left[\partial_{j}-i \hat{A}_{j}, Q\right] \\
& -\frac{1}{8} \sigma_{x y}^{0} \operatorname{Tr} \varepsilon_{i j} Q\left[\partial_{i}-i \hat{A}_{i}, Q\right]\left[\partial_{j}-i \hat{A}_{j}, Q\right] \\
S_{\mathrm{F}}[Q]= & z_{0} \frac{\pi}{\beta}\left[\sum_{n \alpha} \int d^{2} x\left(\operatorname{tr} \mathrm{I}_{n}^{\alpha} Q\right)\left(\operatorname{tr} \mathrm{I}_{-n}^{\alpha} Q\right)+4 \operatorname{Tr} \eta Q-6 \operatorname{Tr} \eta \Lambda\right]
\end{aligned}
$$




$$
\begin{aligned}
S_{\mathrm{U}}[Q, A]= & -\frac{\pi}{\beta} \sum_{n \alpha} \int d^{2} x d^{2} x^{\prime}\left[\operatorname{tr} \mathrm{I}_{n}^{\alpha} Q-\frac{\beta}{\pi}\left(A_{\tau}\right)_{-n}^{\alpha}+\frac{i \beta}{\pi \rho} \sigma_{x y}^{I I} B_{-n}^{\alpha}\right](\vec{x}) U^{-1}\left(\vec{x}, \vec{x}^{\prime}\right) \times \\
& \times\left[\operatorname{tr} \mathrm{I}_{-n}^{\alpha} Q-\frac{\beta}{\pi}\left(A_{\tau}\right)_{n}^{\alpha}+\frac{i \beta}{\pi \rho} \sigma_{x y}^{I I} B_{n}^{\alpha}\right]\left(\vec{x}^{\prime}\right)-\frac{\beta}{4 \pi \rho}\left(\sigma_{x y}^{I I}\right)^{2} \int d^{2} x B^{\dagger} B .
\end{aligned}
$$

The various symbols appearing in this action have the following meaning: The $Q$ and $\mathrm{I}_{n}^{\alpha}$ are matrices carrying combined replica (upper, Greek) and Matsubara frequency (lower, Latin) indices. For instance, the expression $\operatorname{tr} \mathrm{I}_{n}^{\alpha} Q$ stands for $\sum_{k l} \sum_{\beta \gamma}\left(\mathrm{I}_{n}^{\alpha}\right)_{k l}^{\beta \gamma} Q_{l k}^{\gamma \beta}$. The size of all matrices is $2 N_{\max }^{\prime} \times 2 N_{\max }^{\prime}$ in frequency space; the frequency indices run from $-N_{\max }^{\prime}$ to $N_{\max }^{\prime}-1$, while the replica indices run from 1 to $N_{r}$. (See figure 1 for the way in which we sketch matrices in frequency space.) The $Q$-matrix is of the following form,

$$
Q=T^{-1} \Lambda T \quad ; \quad T \in S U(2 N)
$$

where $N=N_{r} \cdot N_{\max }$ with $N_{\max } \ll N_{\max }^{\prime}$ (see figure 1), and $\Lambda$ is given by

$$
\Lambda_{k l}^{\alpha \beta}=\delta^{\alpha \beta}\left[\begin{array}{cc}
1 & 0 \\
0 & -1
\end{array}\right]_{k l}
$$

The $\mathrm{I}_{n}^{\alpha}$ lives in the $\alpha$ 'th replica channel, while in frequency space it is the unity matrix shifted by $n$ places

$$
\left(\mathrm{I}_{n}^{\alpha}\right)_{k l}^{\beta \gamma}=\delta^{\beta \alpha} \delta^{\gamma \alpha} \delta_{k-l, n}
$$

The 'hat' ( $\left.{ }^{\wedge}\right)$ on the external field $A$ in (1.2) denotes a summation with the I-matrices,

$$
\hat{x}:=\sum_{\alpha n} x_{n}^{\alpha} \mathrm{I}_{n}^{\alpha}
$$

The external field is chosen such that $\left(A_{\mu}\right)_{n}^{\alpha}$ is nonzero in the same $n$-interval as $\operatorname{tr} \mathrm{I}_{n}^{\alpha} Q$. The matrix $\eta$ is given by

$$
\eta_{n m}^{\alpha \beta}=n \delta^{\alpha \beta} \delta_{n m}
$$

The Tr stands for a matrix trace combined with spatial integration. The $\sigma_{i j}^{0}$ are the mean field conductances. The $z_{0}$ is the singlet interaction amplitude and $U^{-1}\left(\vec{x}, \vec{x}^{\prime}\right)$ is related to the screened Coulomb interaction. In momentum space $U^{-1}$ is given by

$$
U^{-1}(q)=\frac{\pi / 2}{\rho^{-1}+U_{0}(q)}
$$

with $\rho=d n / d \mu$ the thermodynamic density of states. 


\section{FIGURES}
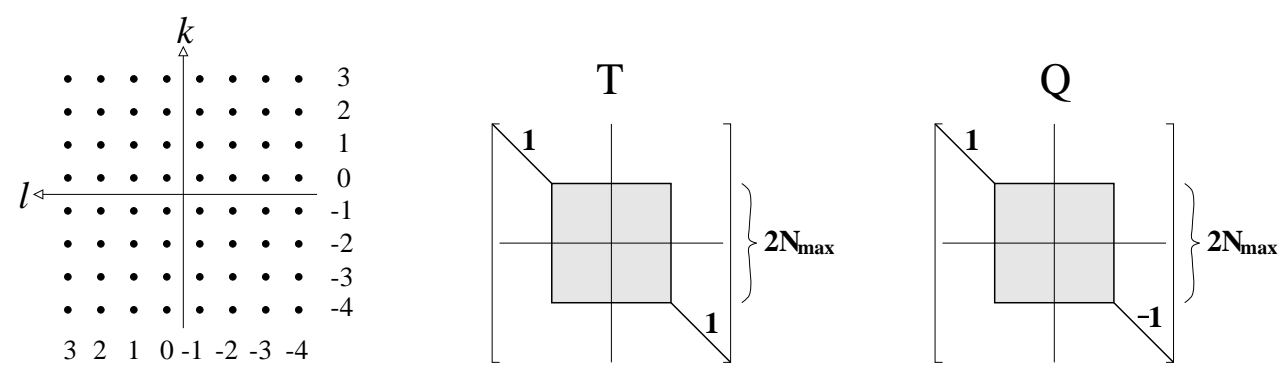

FIG. 1. Sketch of a matrix $[\cdots]_{k l}$ with $k, l$ denoting the Matsubara frequency indices. The 'small' matrices $T$ and $Q$ are nonzero only on the diagonal and within the square of size $2 N_{\max } \times 2 N_{\max }$ (shaded area).
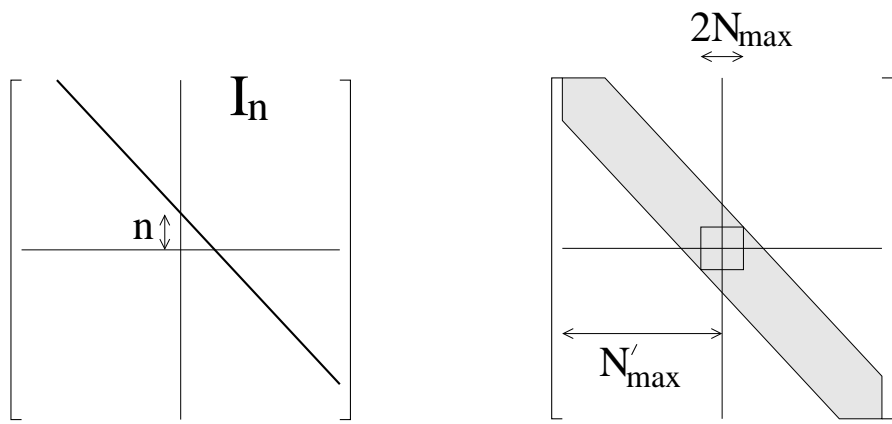

FIG. 2. Left: structure of the 'large' matrix $\mathrm{I}_{n}^{\alpha}(n>0)$. Right: the summation interval $n \in\left\{-2 N_{\max }+1, \cdots, 2 N_{\max }-1\right\}$ is indicated by the shaded area.

\section{Gauge invariance}

Several important remarks have to be made with respect to the gauge invariance of the theory (1.1). At the first stages of the derivation of this action, all matrices were infinite in size $\left(N_{\max }=N_{\max }^{\prime}=\infty\right)$. The infinite $\tilde{I}$-matrices obeyed $\tilde{I}_{n}^{\alpha} \tilde{I}_{m}^{\beta}=\delta^{\alpha \beta} \tilde{I}_{n+m}^{\alpha}$, forming an abelian algebra and generating the electromagnetic gauge transformations,

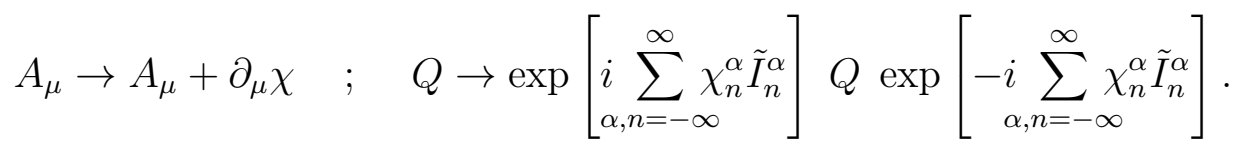

At a certain point the cutoff $N_{\max }^{\prime}$ had to be introduced, destroying the $U(1)$ nature of the $I$-matrices. The truncated $\mathrm{I}_{n}^{\alpha}$ do not even span an algebra any more. The commutators are given by

$$
\left(\mathrm{I}_{n}^{\alpha} \mathrm{I}_{m}^{\beta}\right)_{k l}^{\mu \nu}=\left(\tilde{I}_{n}^{\alpha} \tilde{I}_{m}^{\beta}\right)_{k l}^{\mu \nu} g_{l+m} \quad ; \quad\left[\mathrm{I}_{n}^{\alpha}, \mathrm{I}_{m}^{\beta}\right]_{k l}^{\mu \nu}=\delta^{\alpha \beta \mu \nu} \delta_{k-l, m+n}\left(g_{l+m}-g_{l+n}\right)
$$

where $\delta^{\alpha \beta \mu \nu}$ means that all replica indices have to be the same, and $g_{i}$ is a step function equal to one if $i \in\left\{-N_{\max }^{\prime}, \ldots, N_{\max }^{\prime}-1\right\}$ and zero otherwise. At this stage, there seems to be little hope of preserving any kind of gauge invariance in the theory. 
Disaster is averted in a very subtle way, however. By introducing the second cutoff $N_{\max } \ll N_{\max }^{\prime}$ for the matrix $T$ (and thereby $Q$ ), most of the nasty aspects of the commutations (1.12), living at the edges of Matsubara frequency space, can be avoided. The action (1.1) is invariant under the truncated equivalent of (1.11),

$$
A_{\mu} \rightarrow A_{\mu}+\partial_{\mu} \chi \quad ; \quad Q \rightarrow e^{i \hat{\chi}} Q e^{-i \hat{\chi}} .
$$

The invariance can be checked using the following transformation rules

$$
\begin{aligned}
& \operatorname{tr}\left[\mathrm{I}_{n}^{\alpha} e^{i \hat{\chi}} Q e^{-i \hat{\chi}}\right]=\operatorname{tr} \mathrm{I}_{n}^{\alpha} Q+\frac{\beta}{\pi}\left(\partial_{\tau} \chi\right)_{-n}^{\alpha} \\
& \operatorname{tr}\left[\eta e^{i \hat{\chi}} Q e^{-i \hat{\chi}}\right]=\operatorname{tr} \eta Q-\frac{\beta}{2 \pi} \operatorname{tr} Q \widehat{\partial_{\tau} \chi}-\left(\frac{\beta}{2 \pi}\right)^{2} \sum_{n \alpha}\left(\partial_{\tau} \chi\right)_{-n}^{\alpha}\left(\partial_{\tau} \chi\right)_{n}^{\alpha} .
\end{aligned}
$$

The remarkable aspect of equations (1.14, 1.15) is that they are exact to all powers in $\chi$ as long as $N_{\max } \ll N_{\max }^{\prime}$, and that the cutoff $N_{\max }^{\prime}$ does not appear in them, allowing one to send it safely to infinity.

Using (1.14, 1.15) it is easily seen that $S_{\mathrm{F}}(1.3)$, the 'Finkelstein' part of the action, is by itself invariant under (1.13). This fact is going to be very important in section III. It can also be checked that (1.4) and the two terms in (1.2) are separately invariant. The invariance of the $\sigma_{x y}$-term holds as long as the sample has no boundaries. The effect of a boundary on the theory is very interesting and will be discussed in a subsequent paper.

We will often call the manipulations with the truncated I-matrices by the name of ' $\mathcal{F}$ algebra' and denote the invariance by ' $\mathcal{F}$-invariance'. The invariance of the action does not automatically guarantee invariance of the whole theory; since $Q$ and $e^{i \hat{\chi}} Q e^{-i \hat{\chi}}$ do not

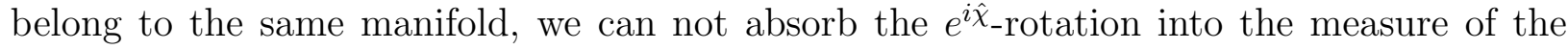
$Q$-integration. The idea behind our approach is, however, that full invariance is regained after sending the cutoff $N_{\max }$ to infinity. In the sections that follow, it is always understood that this limit is taken at the end of all calculations.

\section{BACKGROUND FIELD RENORMALIZATION}

\section{A. The background field method}

Let us start with the action (1.1) without the external fields $A_{\mu}$. We drop the topological term, since it is not going to contribute to perturbation theory. At the moment we are not interested in the full significance of the $S_{\mathrm{U}}$ term, but only in the low-momentum limit. Since $S_{\mathrm{U}}$ really stands for a higher dimensional operator, it is preferable to work with a simpler theory in which the $U^{-1}$ in (1.4) is replaced by a $\delta$-function. This gives, up to a constant,

$$
S[Q]=-\frac{\sigma_{0}}{8} \operatorname{Tr}(\nabla Q)^{2}+\frac{\pi}{\beta} z_{0} \int_{x}\left\{c_{0} \sum_{\alpha n} \operatorname{tr}\left(\mathrm{I}_{n}^{\alpha} Q\right) \operatorname{tr}\left(\mathrm{I}_{-n}^{\alpha} Q\right)+4 \operatorname{tr}(\eta Q)\right\} .
$$

We have introduced a parameter $c_{0}$ such that the theory interpolates between the Coulomb case $\left(c_{0}=1\right)$ and the free particle case $\left(c_{0}=0\right)$. Let us now define

$$
\widehat{Q}=T_{0}^{-1} Q T_{0},
$$


where $T_{0}$ is a 'small' but fixed and slowly varying background field of size, say, $2 n_{\max } \times 2 n_{\max }$, where $n_{\max } \ll N_{\max }$.

If one pursues momentum shell computations then (2.2) is loosely interpreted as a change of variables, where $T_{0}$ stands for the 'slow' modes which should be kept. The $Q$ in (2.2) then really represents the 'fast' modes which should be eliminated. This 'change of variables' idea is clearly somewhat cavalier and complications arise in pursuing the theory beyond one-loop order.

It is the purpose of this section however to show that the basic idea can be put to work and we shall extract important information from it. More specifically, if we keep working with a fixed but 'small' background field, then we can employ the more powerful method of dimensional regularization and compute the effective action for the $T_{0}$-field insertion.

$$
e^{S_{\mathrm{eff}}\left[T_{0}\right]}=\int \mathcal{D} Q e^{S\left[Q, T_{0}\right]}
$$

As was shown in detail in the context of the ordinary $\sigma$ model11, this results in a very effective way of extracting the pole terms in $\varepsilon$, i.e. the renormalization group coefficients $(Z)$ of the theory in $2+2 \varepsilon$ dimensions.

In addition to this we can give precise meaning to the idea of 'small' background field $T_{0}$. In particular, since the $Q$ variables are invariant under a local $U(N) \times U(N)$ transformation $\left(N=N_{\max } \cdot N_{r}\right)$ we may conclude that the abovementioned $S_{\text {eff }}\left[T_{0}\right]$ can be expressed in the local quantity

$$
Q_{0}=T_{0}^{-1} \Lambda T_{0}
$$

This quantity is of the same form as the original variable $Q(1.5)$ except that the sizes $N_{\max }^{\prime}$, $N_{\max }$ are now replaced by $N_{\max }$ and $n_{\max }$ respectively. (See figure 3. .)

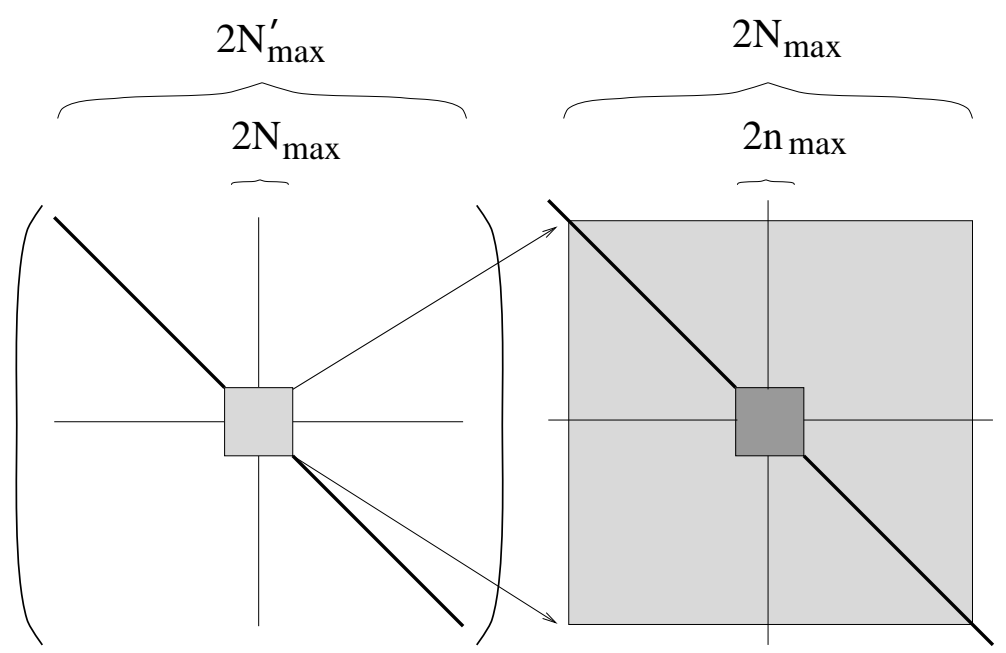

FIG. 3. The relative sizes of $Q$ and the background field matrix $Q_{0}$; see text. 
In different words, the procedure provides the additional information on the renormalization of operators which follows from the requirement that the form of the original action and the form of the shift in the free energy (which we call 'effective action' in $Q_{0}$ ) are the same. Although the basic idea is quite straightforward, it is important to stress that in practice the scheme turns out to be extremely rich and subtle. In order to appreciate the flow of information that can be extracted from it we proceed and describe the results in a step by step fashion. For reasons to be explained shortly, we add a $U(N) \times U(N)$ invariant regulator proportional to $h_{0}^{2}$. The action becomes

$$
\begin{aligned}
S[Q]= & -\frac{\sigma_{0}}{8} \operatorname{Tr}(\nabla Q)^{2}+\frac{\pi}{\beta} z_{0} \int_{x}\left\{c_{0} \sum_{\alpha n} \operatorname{tr}\left(\mathrm{I}_{n}^{\alpha} Q\right) \operatorname{tr}\left(\mathrm{I}_{-n}^{\alpha} Q\right)+4 \operatorname{tr}(\eta Q)\right\} \\
& +\frac{\sigma_{0} h_{0}^{2}}{4} \operatorname{Tr}(\Lambda Q) .
\end{aligned}
$$

One remarkable thing to keep in mind is that the singlet interaction term (proportional to $c_{0}$ ) can not be treated as an operator insertion as one would naively expect. It is, in fact, going to affect the ultraviolet singularity structure of the problem (i.e. poles in $\varepsilon$ ) and, hence, changes the $\beta$-functions of the theory. This eventually happens in the limit where $N_{\max }$ is sent to infinity. This is done in such a way that the quantity $W=T \cdot N_{\max }$ remains finite, and this corresponds physically to to having a finite 'effective' bandwidth $W$.

Next we insert the background field $T_{0}$ in all but the last term of the action. The result can be written as

$$
S\left[Q, T_{0}\right]=S_{k}\left[T_{0}^{-1} Q T_{0}\right]+S_{i}\left[T_{0}^{-1} Q T_{0}\right]+S_{f}\left[T_{0}^{-1} Q T_{0}\right]+\frac{\sigma_{0} h_{0}^{2}}{4} \operatorname{Tr}(\Lambda Q),
$$

where

$$
\begin{aligned}
S_{k}\left[T_{0}^{-1} Q T_{0}\right] & =-\frac{\sigma_{0}}{8} \operatorname{Tr}\left[\partial_{\mu}+A_{\mu}, Q\right]\left[\partial_{\mu}+A_{\mu}, Q\right], \\
S_{i}\left[T_{0}^{-1} Q T_{0}\right] & =\frac{\pi}{\beta} z_{0} c_{0} \int d^{D} x \sum_{\alpha n} \operatorname{tr}\left\{\left(\mathrm{I}_{n}^{\alpha}+A_{n}^{\alpha}\right) Q\right\} \operatorname{tr}\left\{\left(\mathrm{I}_{-n}^{\alpha}+A_{-n}^{\alpha}\right) Q\right\}, \\
S_{f}\left[T_{0}^{-1} Q T_{0}\right] & =\frac{4 \pi}{\beta} z_{0} \int d^{D} x \operatorname{tr}\left\{\left(\eta+A_{\eta}\right) Q\right\} .
\end{aligned}
$$

Here all $T_{0}$ dependence is collected into the 'potentials' $A_{\mu}, A_{n}^{\alpha}$ and $A_{\eta}$

$$
A_{\mu}=T_{0} \partial_{\mu} T_{0}^{-1} \quad A_{m}^{\alpha}=T_{0}\left[\mathrm{I}_{m}^{\alpha}, T_{0}^{-1}\right] \quad A_{\eta}=T_{0}\left[\eta, T_{0}^{-1}\right] .
$$

The effective action $S_{\text {eff }}\left[T_{0}\right]$ is going to be obtained by treating the 'potentials' $A_{\mu}, A_{m}^{\alpha}$ and $A_{\eta}$ as a perturbation about the original action (2.5). It is easy to see however, that this procedure breaks the $U(N) \times U(N)$ gauge invariance which means that the final result can not be expressed, generally speaking, in terms of the local variable $Q_{0}$ (2.4). In order to retain $U(N) \times U(N)$ gauge invariance it will be necessary to drop all temperature and frequency dependence as infrared regulators such that the parameters in the effective theory only depend on the regulating field $h_{0}^{2}$. We will come back to this problem at a later stage (section [ID). 


\section{B. Perturbation expansion}

The theory (2.6) is going to be worked out perturbatively. Write

$$
Q=\left(\begin{array}{cc}
\sqrt{1-q q^{\dagger}} & q \\
q^{\dagger} & -\sqrt{1-q^{\dagger} q}
\end{array}\right),
$$

then the theory can be written as an infinite power series in the matrix fields $q, q^{\dagger}$ which contain $N \times N$ independent complex field variables. Notice that classically (i.e. $Q=\Lambda$ ) the effective action $S\left[\Lambda, T_{0}\right]$ is of the same form as the original one (2.5) except that the $\operatorname{Tr}(\Lambda Q)$ term is lacking.

In order to discuss the theory on a quantum level we regroup the various quantities in the background field action (2.6) according to

$$
\begin{aligned}
S\left[Q, T_{0}\right]= & S[Q]+S\left[T_{0}^{-1} \Lambda T_{0}\right]-\int d^{D} x\left\{\frac{\sigma_{0}}{4} \cdot O_{k}\left(Q, T_{0}\right)-\frac{4 \pi}{\beta} z_{0} \cdot O_{f}\left(Q, T_{0}\right)\right. \\
& \left.-\frac{\pi}{\beta} z_{0} c_{0} \cdot O_{i}\left(Q, T_{0}\right)\right\}
\end{aligned}
$$

where $O_{k}=O_{k}^{(1)}+O_{k}^{(2), 1}+O_{k}^{(2), 2} \quad ; \quad O_{i}=O_{i}^{(1), 1}+O_{i}^{(1), 2}+O_{i}^{(2), 1}+O_{i}^{(2), 2}$. The eight different operators $O$ are listed in Table 1.

\begin{tabular}{|c||c|}
\hline$O_{k}^{(1)}=2 \operatorname{tr}\left(A_{\mu} \delta Q \partial_{\mu} \delta Q\right)$ & $O_{i}^{(1), 1}=2 \sum_{m, \alpha} \operatorname{tr}\left(\mathrm{I}_{-m}^{\alpha} Q_{0}\right) \operatorname{tr}\left(\mathrm{I}_{m}^{\alpha} \delta Q\right)$ \\
\hline$O_{k}^{(2), 1}=2 \operatorname{tr}\left(\delta Q A_{\mu} \Lambda A_{\mu}\right)$ & $O_{i}^{(1), 2}=2 \sum_{m, \alpha} \operatorname{tr}\left(\mathrm{I}_{-m}^{\alpha} \delta Q\right) \operatorname{tr}\left(A_{m}^{\alpha} \delta Q\right)$ \\
\hline$O_{k}^{(2), 2}=\operatorname{tr}\left(A_{\mu} \delta Q A_{\mu} \delta Q\right)$ & $O_{i}^{(2), 1}=2 \sum_{m, \alpha} \operatorname{tr}\left(\mathrm{I}_{-m}^{\alpha} Q_{0}\right) \operatorname{tr}\left(A_{m}^{\alpha} \delta Q\right)$ \\
\hline$O_{f}=\operatorname{tr}\left(A_{\eta} \delta Q\right)$ & $O_{i}^{(2), 2}=\sum_{m, \alpha} \operatorname{tr}\left(A_{-m}^{\alpha} \delta Q\right) \operatorname{tr}\left(A_{m}^{\alpha} \delta Q\right)$ \\
\hline
\end{tabular}

Table 1: Classification of terms in $\delta Q=Q-\Lambda$ that contribute to the background field action; see text.

The superscript (in brackets) of $O$ shows the lowest order of $\delta T_{0}=T_{0}-1$ which appears in the decomposition of the expression in powers of $\delta T_{0}$, while the other one (if present) just indicates the enumeration of such a contribution. The propagator for the $q$-field can be read from the quadratic part of the action (2.5) and has the form

$$
\left\langle q_{n_{1} n_{2}}^{\alpha \beta}(p)\left[q^{\dagger}\right]_{n_{4} n_{3}}^{\delta \gamma}(-p)\right\rangle=\frac{4}{\sigma_{0}} \delta^{\alpha \gamma} \delta^{\beta \delta} \delta_{n_{1}-n_{2}, n_{3}-n_{4}} D_{p}\left(n_{12}\right)\left\{\delta_{n_{1} n_{3}}+\delta^{\alpha \beta} \kappa^{2} z_{0} c_{0} D_{p}^{c}\left(n_{12}\right)\right\},
$$




$$
\begin{array}{cll}
D_{p}\left(n_{12}\right)=\frac{1}{p^{2}+h_{0}^{2}+\kappa^{2} z_{0} n_{12}} \quad ; \quad & D_{p}^{c}\left(n_{12}\right)=\frac{1}{p^{2}+h_{0}^{2}+\left(1-c_{0}\right) \kappa^{2} z_{0} n_{12}} \\
\kappa^{2}:=\frac{8 \pi}{\beta \sigma_{0}} \quad ; \quad n_{12}:=n_{1}-n_{2} .
\end{array}
$$

Here and in what follows we use the following convention: Matsubara indices with odd subscripts $\left(n_{1}, n_{3}, \ldots\right)$ run only over nonnegative values, while those with even subscripts $\left(n_{2}, n_{4}, \ldots\right)$ run only over negative values. This choice is made in order to incorporate the structure (2.11), where it is clear that the first and second index of $q$ have to be nonnegative and negative respectively. (And vice versa for $q^{\dagger}$ ). Often we use the abbreviation

$$
\alpha=1-c_{0} .
$$

In the case of Coulomb interactions we have $\alpha \rightarrow 0$.

In the following sections we present the results of a detailed computation that shows that the final results for $S_{\text {eff }}$ are of the same form as the original action (2.5) with, however, modified 'effective' parameters $\sigma^{\prime}, z^{\prime}$ and $c^{\prime}$ instead of $\sigma_{0}, z_{0}$ and $c_{0}$.

\section{Conductivity renormalization}

The contributions to the conductivity $\sigma^{\prime}$ have four different sources. Schematically, they come from the contractions in the following terms: $\left\langle O_{k}^{(2), 1}\right\rangle,\left\langle\left[O_{k}^{(1)}\right]^{2}\right\rangle,\left\langle\left[O_{i}^{(1), 2}\right]^{2}\right\rangle$ and $\left\langle O_{k}^{(1)} O_{i}^{(1), 2}\right\rangle$, with the quantities $O_{k}$ and $O_{i}$ defined in Table 1. The last two of these contributions involve a small momentum expansion in the background field $T_{0}$. In Table 2 we present the various contributions to the pole terms in $1 / \varepsilon$ obtained by working in $D=2+2 \varepsilon$ dimensions and using dimensional regularization.

\begin{tabular}{|c|c|c|c|c|}
\hline & $\left\langle O_{k}^{(2), 1}\right\rangle$ & $-\frac{1}{2}\left\langle\left[O_{k}^{(1)}\right]^{2}\right\rangle$ & $-\frac{1}{2}\left\langle\left[O_{i}^{(1), 2}\right]^{2}\right\rangle$ & $-\left\langle O_{k}^{(1)} O_{i}^{(1), 2}\right\rangle$ \\
\hline $\operatorname{tr}\left(A_{\mu}^{+-} A_{\mu}^{-+}+A_{\mu}^{-+} A_{\mu}^{+-}\right)$ & $\ln \alpha$ & 0 & $-\left(2+\frac{1+\alpha}{1-\alpha} \ln \alpha\right)$ & 0 \\
\hline $\operatorname{tr}\left(A_{\mu}^{++} A_{\mu}^{++}+A_{\mu}^{--} A_{\mu}^{--}\right)$ & $-\ln \alpha$ & $-2\left(1+\frac{\alpha}{1-\alpha} \ln \alpha\right)$ & $-\left(2+\frac{1+\alpha}{1-\alpha} \ln \alpha\right)$ & $2\left(2+\frac{1+\alpha}{1-\alpha} \ln \alpha\right)$ \\
\hline
\end{tabular}

Table 2: Contributions from different sources (top row) to the $1 / \varepsilon$ pole terms in the background field action (left column); see text.

The most important terms generated by the background field procedure are $\left(A_{\mu}\right)_{n_{1} n_{2}}^{\alpha \beta}\left(A_{\mu}\right)_{n_{2} n_{1}}^{\beta \alpha}$, which are indicated by $\operatorname{tr} A_{\mu}^{+-} A_{\mu}^{-+}$in Table 2 , the $\left(A_{\mu}\right)_{n_{1} n_{3}}^{\alpha \beta}\left(A_{\mu}\right)_{n_{3} n_{1}}^{\beta \alpha}$, which are denoted as $\operatorname{tr} A_{\mu}^{++} A_{\mu}^{++}$and finally those obtained by interchanging the positive and negative Matsubara frequency indices. Notice that the first terms can be written in $\sigma$-model form

$$
\operatorname{tr}\left(A_{\mu}^{+-} A_{\mu}^{-+}+A_{\mu}^{-+} A_{\mu}^{+-}\right)=-\frac{1}{4} \operatorname{tr}\left(\nabla Q_{0}\right)^{2}
$$


The terms on the second line of Table 2 can not be written in terms of the quantity $Q_{0}$ as they break the local $U\left(N_{\max }\right) \times U\left(N_{\max }\right)$ gauge invariance. The various contributions in this case sum up to zero, however, such that the final theory retains the $U\left(N_{\max }\right) \times U\left(N_{\max }\right)$ symmetry. The final result for the effective parameter $\sigma^{\prime}$ becomes

$$
\sigma^{\prime}=\sigma_{0}\left(1+\frac{4 h_{0}^{2 \varepsilon}}{\sigma_{0} \varepsilon} \Omega_{D}\left[1+\frac{\alpha}{1-\alpha} \ln \alpha\right]\right)
$$

where $\Omega_{D}=\frac{S_{D}}{2(2 \pi)^{D}}$ with $S_{d}$ the surface of the unit sphere in $d$ dimensions. Notice that (2.16) behaves smoothly as $\alpha$ approaches zero (Coulomb case) whereas the individual contributions listed in Table 2 do not. On the other hand, (2.16) reduces to the well known free particle case as $\alpha \rightarrow 1$. In what follows, it will be convenient to introduce the inverse conductivity $t_{0}$

$$
t_{0}=4 \Omega_{D} / \sigma_{0} .
$$

We can remove the pole term in $\varepsilon$ from (2.16) by defining a renormalized theory $t_{0}=\mu^{-2 \varepsilon} t Z_{1}$ as usual, leading to a finite expression in $\varepsilon$. From (2.16) we extract

$$
Z_{1}=1+\frac{t}{\varepsilon}\left[1+\frac{\alpha}{1-\alpha} \ln \alpha\right]
$$

yielding

$$
\frac{1}{4 \Omega_{D}} \sigma^{\prime}=t^{-1}\left(1+t \ln \left(\mu^{2} h_{0}^{2}\right)\left[1+\frac{\alpha}{1-\alpha} \ln \alpha\right]\right) .
$$

Notice that the parameter $h_{0}$ appears as an infrared cutoff rather than the frequency or temperature, and here it plays the role of the (inverse) sample size. In the next section we give an explicit example indicating the kind of complications one is running into by working with finite temperature or frequency rather than $h_{0}$.

We now have all the necessary ingredients to calculate the beta function for the conductance,

$$
\beta=\frac{d t}{d \ln \mu}=2 \varepsilon t-2 t^{2}\left[1+\frac{\alpha}{1-\alpha} \ln \alpha\right]
$$

\section{Frequency and interaction renormalization}

The singular contributions corresponding to the terms quadratic in $A_{m}$ and $A_{\eta}$ (2.10) in $S_{\text {eff }}$ originate from many sources. They are contained in the following contractions: $\left\langle O_{f}\right\rangle,\left\langle O_{i}^{(1), 2}\right\rangle,\left\langle O_{i}^{(2), 1}\right\rangle,\left\langle O_{i}^{(2), 2}\right\rangle,-\frac{1}{2}\left\langle\left[O_{i}^{(1), 2}\right]^{2}\right\rangle$ and $-\left\langle O_{i}^{(1), 1} O_{i}^{(1), 2}\right\rangle$, while all other possible sources give answers which are finite in $\varepsilon$. Each of the individual contractions gives a divergent answer as $\alpha$ approaches zero, just as in the computation of $\sigma^{\prime}$. However, numerous cancelations of these divergencies take place in the total sum. The resulting expression for $S_{\text {eff }}\left[T_{0}\right]$ is given by

$$
\begin{aligned}
& -\frac{\pi}{\beta} z_{0}\left\langle 4 O_{f}+2 c_{0} O_{i}^{(1), 2}+2 c_{0} O_{i}^{(2), 1}+c_{0} O_{i}^{(2), 2}-\frac{\pi}{\beta} z_{0} c_{0}^{2}\left[O_{i}^{(1), 2}\right]^{2}-\frac{2 \pi}{\beta} z_{0} c_{0}^{2} O_{i}^{(1), 1} O_{i}^{(1), 2}\right\rangle \\
& \longrightarrow \Gamma_{0}+\sum_{\alpha \beta \gamma} \sum_{m n_{1} n_{2}}^{\prime} \Gamma_{1}\left(m, n_{1}, n_{2}\right)\left(T_{0} \mathrm{I}_{m}^{\alpha} T_{0}^{-1}\right)_{n_{2} n_{1}}^{\beta \gamma}\left(T_{0} \mathrm{I}_{-m}^{\alpha} T_{0}^{-1}\right)_{n_{1} n_{2}}^{\gamma \beta}
\end{aligned}
$$


with $\Gamma_{1}$ defined as

$$
\Gamma_{1}\left(m, n_{1}, n_{2}\right)=-\kappa^{2} z_{0} c_{0} \int_{p} D_{p}\left(n_{12}\right)\left[1+\kappa^{2} z_{0} c_{0}|m| D_{p}^{c}(|m|)\right]
$$

and where the prime on the frequency summation indicates a restriction on the frequency

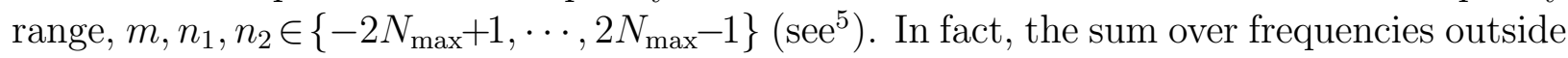
of this range cancels the contribution from $\left\langle O_{f}\right\rangle$.

In (2.21) we have included a constant $\Gamma_{0}$ which is such that it cancels the constant that appears in the $\Gamma_{1}$ term. The singular contribution is obtained by replacing the square bracket in (2.22) by unity, and we get simply

$$
\int_{p} D_{p}\left(n_{12}\right)=-\frac{\Omega_{D}}{\varepsilon}\left(h_{0}^{2}+\kappa^{2} z_{0} n_{12}\right)^{\varepsilon} \approx-\frac{\Omega_{D}}{\varepsilon}-\Omega_{D} \ln \left(h_{0}^{2}+\kappa^{2} z_{0} n_{12}\right) .
$$

We mention two important aspects of this result. First, the pole term in $\varepsilon$ is independent of the frequency indices $n_{1}, n_{2}$ and this implies that the result (2.21) becomes $U\left(N_{\max }\right) \times U\left(N_{\max }\right)$ invariant, i.e. it can be expressed in the quantity $Q_{0}$ (see below). Notice that $U\left(N_{\max }\right) \times$ $U\left(N_{\max }\right)$ gauge invariance is automatically obtained by putting the ratio $\kappa^{2} z_{0} n_{12} / h_{0}^{2}$ equal to zero, i.e. by dropping the temperature and frequency as infrared regulator in the final answer. This, however, is just a different way of saying that the background field procedure, along with any other arbitrary RG program, can never provide information on the dynamics of the problem, such as the AC conductivity.

Secondly, we evaluate the result (2.21) as follows.

$$
\begin{aligned}
S_{\text {eff }}\left[T_{0}\right] & =\Gamma_{0}-\kappa^{2} z_{0} c_{0} \frac{h_{0}^{2 \varepsilon}}{\varepsilon} \Omega_{D} \sum_{\alpha \beta \gamma} \sum_{m n_{1} n_{2}}^{\prime}\left(T_{0} \mathrm{I}_{m}^{\alpha} T_{0}^{-1}\right)_{n_{2} n_{1}}^{\beta \gamma}\left(T_{0} \mathrm{I}_{-m}^{\alpha} T_{0}^{-1}\right)_{n_{1} n_{2}}^{\gamma \beta} \\
& =\Gamma_{0}+\kappa^{2} z_{0} c_{0} \frac{h_{0}^{2 \varepsilon}}{8 \varepsilon} \Omega_{D} \sum_{m \alpha}^{\prime} \operatorname{tr}\left[T_{0} \mathrm{I}_{m}^{\alpha} T_{0}^{-1}, \Lambda\right]\left[T_{0} \mathrm{I}_{-m}^{\alpha} T_{0}^{-1}, \Lambda\right] \\
& =\Gamma_{0}+\kappa^{2} z_{0} c_{0} \frac{h_{0}^{2 \varepsilon}}{8 \varepsilon} \Omega_{D} \sum_{m \alpha}^{\prime} \operatorname{tr}\left[\mathrm{I}_{m}^{\alpha}, Q_{0}\right]\left[\mathrm{I}_{-m}^{\alpha}, Q_{0}\right]
\end{aligned}
$$

which has precisely the $\mathcal{F}$-invariant form in $Q_{0}$ given in $(1.3)$. Hence, the significance of working with 'small' background fields $T_{0}$ (relative to the field variables $Q$ ) is now well recognized and the renormalization procedure for the interacting electron gas involves not only the usual rescaling of momenta, it necessarily involves also a rescaling of the time or frequency variable or, rather, of the 'effective' bandwidth $W=T N_{\max }$.

Next, from (2.24) we can read off the numerical value for the constant $\Gamma_{0}$ which is given by

$$
\Gamma_{0}=\frac{3}{2} \kappa^{2} z_{0} c_{0} \frac{h_{0}^{2 \varepsilon}}{\varepsilon} \Omega_{D} N_{r} \sum_{m}|m|
$$

It can be shown (Section IVB) that $\Gamma_{0}$ is precisely canceled by the one-loop result for the free energy $\left(\mathcal{F}_{1}\right)$ which still appears in the definition of $S_{\text {eff }}$. Hence the expression in $Q_{0}$ (2.24) is an eigenoperator of the renormalization procedure, and this is in sharp contrast to what one is used to in the conventional $\sigma$-model theory applied to the free electron problem 13 . Operators bilinear in $Q$ as well as higher order operators in this case describe 
density fluctuations which become anomalous (multifractal) as one approaches the critical point (mobility edge) in $2+2 \varepsilon$ dimensions. The physics of the interacting electron gas appears to be quite different in this respect and, as will be shown in later sections, the theory is much closer to the familiar one describing the Heisenberg ferromagnet. In particular, the interacting system is characterized by a conventional order parameter, as well as a (Coulomb) gap in the quasiparticle density of states which enters the expression for the specific heat.

We summarize the results of this section by giving the complete form for the effective background field action (including constants)

$$
\begin{aligned}
S_{\text {eff }}\left[Q_{0}\right]= & -\frac{\sigma^{\prime}}{8} \operatorname{Tr}\left(\nabla Q_{0}\right)^{2}+\frac{\pi}{2 \beta} z^{\prime} \sum_{\alpha n}^{\prime} \operatorname{Tr}\left[\mathrm{I}_{n}^{\alpha}, Q_{0}\right]\left[\mathrm{I}_{-n}^{\alpha}, Q_{0}\right] \\
& +\frac{\pi}{\beta} \alpha^{\prime} z^{\prime} \int_{x} \sum_{n \alpha} \operatorname{tr} \mathrm{I}_{n}^{\alpha} Q_{0} \operatorname{tr} \mathrm{I}_{-n}^{\alpha} Q_{0}
\end{aligned}
$$

where the effective parameters $\sigma^{\prime}, c^{\prime}=1-\alpha^{\prime}$ and $z^{\prime}$ are given by $(2.16)$ and

$$
\begin{aligned}
z^{\prime} & =z_{0}\left(1+\frac{2}{\sigma_{0}} c_{0} \Omega_{D} \frac{h_{0}^{2 \varepsilon}}{\varepsilon}\right)=z_{0}\left(1+\frac{h_{0}^{2 \varepsilon}}{2 \varepsilon} c_{0} t_{0}\right) \\
\alpha^{\prime} z^{\prime} & =\left(1-c^{\prime}\right) z^{\prime}=\left(1-c_{0}\right) z_{0}=\alpha_{0} z_{0} .
\end{aligned}
$$

Introducing renormalization constants for the amplitudes, $z_{0}=\mu^{2 \varepsilon} z Z_{2}$ and $\alpha_{0}=\alpha Z_{\alpha}$, we then extract from (2.27)

$$
Z_{\alpha}=Z_{2}^{-1} \quad ; \quad Z_{2}=1-\frac{t}{2 \varepsilon} c
$$

such that the renormalization of the $\alpha$ is obtained as

$$
\frac{d \alpha}{d \ln \mu}=-t \alpha(1-\alpha)
$$

and the anomalous dimension of the amplitude $z$ is expressed in terms of the $\gamma$-function

$$
\gamma=-\frac{d\left(\mu^{2 \varepsilon} z\right)}{d \ln \mu}=\frac{d \ln Z_{2}}{d \ln \mu}=-t(1-\alpha)
$$

Equation (2.29) implies that $\mathcal{F}$-invariance of the theory $(\alpha=0)$ is retained by the renormalization group. The theory is unstable with respect to the symmetry breaking $\alpha$ which plays a role analogous to the 'range' of the electron-electron interactions. From the $\beta$-function (2.20) and (2.30) we conclude that the Coulomb interaction problem $(\alpha=0)$ has a fixed point $t_{c} \propto \varepsilon$ in $2+2 \varepsilon$ dimensions which separates a metallic phase $\left(t<t_{c}\right)$ from an insulating phase $\left(t>t_{c}\right)$. In two spatial dimensions, the metallic phase disappears altogether and this, then, leads to the familiar complications as far as the quantum Hall effect is concernedi.

\section{LINEAR RESPONSE}

In the previous section we have seen that within the background field approach, the singlet interaction term contributes in a peculiar fashion to the conductivity renormalization. In this section we present the results of a detailed computation of $\sigma^{\prime}$ by considering the linear response to a vector potential insertion. If one views the insertion of a vector potential as 
the result of a ('large') background field rotation $W$, then it is clear that this special type of background field leaves the Finkelstein action invariant, the symmetry being broken only by $\alpha \neq 0$ for $h_{0}^{2}=0$.

In contrast to the background field procedure, we can now expect that the limit $h_{0} \rightarrow 0$ can be taken in the end such that the frequency and $T$ dependence of the $\sigma^{\prime}$ can be computed. We stress that it is a priori completely un-obvious that the two different ways of computing $\sigma^{\prime}$ should yield identical renormalization group results in the end. In fact, the different methods correspond to two completely different manners of handling the small frequency, long wavelength excitations of the system. In a previous paper we have shown that these different methods are formally related by the $U\left(N_{\max }\right) \times U\left(N_{\max }\right)$ gauge invariance of the theory. Hence, the results of this section should be considered as an important demonstration of the significance of the $\mathcal{F}$-invariance present in the problem.

We start out by replacing the derivatives in (2.5) by covariant derivatives

$$
\partial_{j} Q \rightarrow\left[\partial_{j}-i \hat{A}_{j}, Q\right]
$$

The 'effective' action for the vector potential $\vec{A}$ is defined by

$$
e^{S_{\mathrm{eff}}[\vec{A}]}=\int \mathcal{D}[Q] e^{S[Q, \vec{A}]}
$$

which is generally given as

$$
S_{\mathrm{eff}}[\vec{A}]=\int_{x} \sum_{\alpha, n>0} \sigma^{\prime}(n) n\left[\vec{A}_{n}^{\alpha}\right]^{*} \cdot \vec{A}_{n}^{\alpha}
$$

The unknown quantity $\sigma^{\prime}(n)$ can be formally expressed in terms of correlations of the $Q$-fields as follows

$$
\begin{aligned}
\sigma^{\prime}(n)= & -\frac{\sigma_{0}}{4 n} \operatorname{tr}\left\langle\left[\mathrm{I}_{n}^{\alpha}, Q\right]\left[\mathrm{I}_{-n}^{\alpha}, Q\right]\right\rangle \\
& +\frac{\sigma_{0}^{2}}{16 n D} \int_{x^{\prime}}\left\langle\left\{\operatorname{tr}\left[\mathrm{I}_{n}^{\alpha} Q\right] \nabla Q(x)\right\} \cdot\left\{\operatorname{tr}\left[\mathrm{I}_{-n}^{\alpha} Q\right] \nabla Q\left(x^{\prime}\right)\right\}\right\rangle
\end{aligned}
$$

where the expectation is with respect to the theory of (2.5). Here we take a single fixed replica channel. At a classical level $(Q=\Lambda)$, equation (3.4) gives us $\sigma^{\prime}=\sigma_{0}$ as it should. The quantum fluctuations of the $Q$-field give rise to various contributions which are individually singular as $\alpha$ approaches zero. A detailed computation gives the following answer

$$
\begin{aligned}
\sigma^{\prime}(n)= & \sigma_{0}-\frac{16 \kappa^{4} c_{0}}{D} \frac{2 \pi}{\beta} z_{0}^{2} \sum_{m=1}^{\infty} m \int_{p} p^{2} D_{p}(m+n) D_{p}(m+2 n) D_{p}^{c}(m) \times \\
& \times\left[D_{p}(m)+\frac{1-c_{0}}{2} D_{p}^{c}(m+n)\right] .
\end{aligned}
$$

The sum and integral can be performed by keeping one of the regulators $h_{0}^{2}$, temperature or frequency $\omega=\frac{2 \pi}{\beta} n$ fixed and putting the other two to zero. The general result involves complex expressions containing dilogs and $\psi$-functions. Here we limit the discussion to the most interesting case of the Coulomb interaction $\left(\alpha=1-c_{0}=0\right)$. We find to lowest order in $\varepsilon$ 


$$
\begin{aligned}
\sigma^{\prime}(T) & =\sigma_{0}-4 \Omega_{D}\left(-\frac{1}{\varepsilon}-\ln \left(\frac{8 \pi}{\beta \sigma_{0}} z_{0}\right)+2+\gamma\right) \\
\sigma^{\prime}(\omega) & =\sigma_{0}-4 \Omega_{D}\left(-\frac{1}{\varepsilon}-\ln \frac{z_{0} \omega}{\sigma_{0}}+\frac{3}{2}-2 \ln 2\right) \\
\sigma^{\prime}\left(h_{0}^{2}\right) & =\sigma_{0}-4 \Omega_{D}\left(-\frac{1}{\varepsilon}-\ln h_{0}^{2}\right) .
\end{aligned}
$$

Here, $\gamma \approx 0.5772 \ldots$ denotes Euler's constant. As before, the numerical factor $\Omega_{D}$ can be absorbed in a redefinition of the parameters $\sigma^{\prime}, \sigma_{0}$. Notice that (3.8) is precisely the result we previously obtained by employing the background field procedure. A good approximation to the general case is obtained by replacing $\ln h_{0}^{2}$ in (3.8) by the following expression with appropriately chosen constants $a$ and $b$

$$
\ln h_{0}^{2} \rightarrow \ln \left[h_{0}^{2}+a \frac{z_{0} \omega}{\sigma_{0}}+b \frac{z_{0} T}{\sigma_{0}}\right] .
$$

This expression indicates that the square of the phase breaking length $\left(L_{\varphi}^{2}\right)$ due to the different mechanisms of sample size, frequency and temperature add up in parallel. We have convinced ourselves of this result by performing numerical analyses.

\section{FREE ENERGY}

\section{A. Introduction}

In this section we build upon an important result obtained from the background field procedure. In particular the construction of the eigenoperators (which includes the constants in the quantum fluctuations $q, q^{\dagger}$ ) can be exploited in a very effective fashion by extracting higher loop renormalization results from a computation of the free energy analogy with what has been done for ordinary $\sigma$-models 2 . However, the computation of the free energy in our case provides additional information not only on the theory in strong coupling, it also gives rise to the identification of a new physical quantity in the problem, namely the quasiparticle density of states entering the specific heat.

Since the apparatus of the renormalization group can only be put to work once the singularity structure of the free theory is known, we shall proceed by first reporting the results of a detailed computation of the free energy to two-loop order. These results indicate that the quantum theory retains the tree level structure of the free energy, a result which is obtained by putting $Q_{0}=\Lambda$ in our background field functional (2.26). More specifically, write

$$
S_{\mathrm{eff}}\left[Q_{0}=\Lambda\right]=-2 V_{D} N_{r} \sum_{n>0}^{\prime} z^{\prime} \omega_{n}
$$

where the prime on the summation sign now merely indicates that the limit of small frequencies $\omega_{n}=\frac{2 \pi}{\beta} n$ is of interest only. The $V_{D}$ stands for the volume of the $D$-dimensional system.

For reasons which have been well explained in sections $\mathbb{\text { II }}$ and III, the background field procedure only provides information on the quantity $z^{\prime}$ in the case where the $h_{0}^{2}$ provides the dominant infrared regularization. Since $\mathcal{F}$-invariant quantities like the free energy are, in principle, free of such a constraint, it is important to know whether and how the regulator 
$h_{0}^{2}$ can be put equal to zero. Most of the answer to the question is already provided by the theory in one-loop approximation which will be discussed in section IVB. In section IVG we report the results of the much more complicated theory at two-loop level. The reader who is not interested in technical details might skip this section and proceed immediately to section $\nabla$ where the scaling implications are analyzed, making use (amongst other things) of the more familiar theory of the Heisenberg ferromagnet.

\section{B. One-loop theory}

Let us proceed from the results obtained from the background field procedure (2.26) and start from the renormalizable action

$$
S[Q]=-\frac{\sigma_{0}}{8} \operatorname{Tr}(\nabla Q)^{2}+\frac{\pi}{2 \beta} z_{0} \sum_{\alpha n}^{\prime} \operatorname{Tr}\left[\mathrm{I}_{n}^{\alpha}, Q\right]\left[\mathrm{I}_{-n}^{\alpha}, Q\right]-\frac{\pi}{\beta} z_{0} \alpha_{0} \sum_{n \alpha} \int_{x} \operatorname{tr} \mathrm{I}_{n}^{\alpha} Q \operatorname{tr} \mathrm{I}_{-n}^{\alpha} Q .
$$

This action can be written in a form where the 'large' diagonal components of $Q$ do not contribute,

$$
\begin{aligned}
S[Q]= & -2 z_{0} V_{D} N_{r} \sum_{n>0}^{\prime} \omega_{n}-\frac{\sigma_{0}}{8} \operatorname{Tr}(\nabla Q)^{2}+\frac{\pi}{\beta} z_{0} c_{0} \sum_{n \alpha} \int_{x} \operatorname{tr} \mathrm{I}_{n}^{\alpha} Q \operatorname{tr} \mathrm{I}_{-n}^{\alpha} Q \\
& +4 z_{0} \frac{\pi}{\beta} \operatorname{Tr} \eta(Q-\Lambda) .
\end{aligned}
$$

The free energy $\mathcal{F}$ is defined by

$$
e^{-V_{D} N_{r} \mathcal{F}}=\int \mathcal{D}[Q] e^{S[Q]}
$$

and can be written as

$$
\mathcal{F}=\mathcal{F}_{0}+\mathcal{F}_{1}+\mathcal{F}_{2}+\cdots
$$

where the subscript $i$ on $\mathcal{F}_{i}$ indicates the 'order' of the loop expansion. The quantity $\mathcal{F}_{0}$ is given by the first term in (4.3),

$$
\mathcal{F}_{0}=2 z_{0} \sum_{n>0}^{\prime} \omega_{n}
$$

On the other hand, the $\mathcal{F}_{1}$ is readily obtained from the Gaussian quantum theory and the result is

$$
\begin{aligned}
\mathcal{F}_{1} & =\sum_{n>0} \int_{p} \ln \frac{p^{2}+h_{0}^{2}+\alpha \kappa^{2} z_{0} n}{p^{2}+h_{0}^{2}+\kappa^{2} z_{0} n} \\
& =-\Omega_{D} \sum_{n>0} \frac{\left(h_{0}^{2}+\kappa^{2} z_{0} n\right)^{1+\varepsilon}-\left(h_{0}^{2}+\alpha \kappa^{2} z_{0} n\right)^{1+\varepsilon}}{\varepsilon(1+\varepsilon)} .
\end{aligned}
$$

By taking the $\ln T$-derivative of $\mathcal{F}$ one reproduces the result of the background field procedure (4.1), 


$$
\frac{\partial \mathcal{F}}{\partial \ln T}=2 \sum_{n>0}^{\prime} z_{0} \omega_{n}\left\{1+\frac{2 \Omega_{D}}{\sigma_{0}} \frac{\left(h_{0}^{2}+\kappa^{2} z_{0} n\right)^{\varepsilon}-\alpha\left(h_{0}^{2}+\alpha \kappa^{2} z_{0} n\right)^{\varepsilon}}{\varepsilon}\right\} .
$$

By neglecting the $\omega_{n}$ terms relative to the infrared regulator $h_{0}^{2}$, one obtains

$$
\frac{\partial \mathcal{F}}{\partial \ln T}=2 z^{\prime} \sum_{n>0}^{\prime} \omega_{n}
$$

with $z^{\prime}$ precisely given by the background field result of (4.1). Next we consider (4.8) in the limit $h_{0}^{2} \rightarrow 0$. In this case (4.8) defines a $\omega_{n}$-dependent effective parameter $z^{\prime}\left(\omega_{n}\right)$,

$$
\frac{\partial \mathcal{F}}{\partial \ln T}=2 \sum_{n>0}^{\prime} z^{\prime}\left(\omega_{n}\right) \omega_{n}
$$

where

$$
z^{\prime}\left(\omega_{n}\right)=z_{0}\left\{1+\frac{2 \Omega_{D}}{\sigma_{0}}\left(\frac{4 z_{0} \omega_{n}}{\sigma_{0}}\right)^{\varepsilon} \frac{1-\alpha^{1+\varepsilon}}{\varepsilon}\right\}
$$

\section{Two-loop theory}

Treating the nonquadratic part of the Hamiltonian as a perturbation, the expression for the two-loops contribution to the free energy can be presented in the form

$$
\mathcal{F}_{2}=-\left\langle S_{0}^{(4)}+S_{\text {int }}^{(4)}+\frac{1}{2}\left(S_{\text {int }}^{(3)}\right)^{2}\right\rangle
$$

where the superscript between brackets denotes the order in $q, q^{\dagger}$. The $S_{0}$ stands for the 'free' action without the interaction term, and its fourth order part is given by

$$
\begin{aligned}
S_{0}^{(4)}= & \frac{\sigma_{0}}{32} \int_{p} \sum q_{n_{1} n_{2}}^{\alpha \beta}\left(p_{1}\right)\left[q_{n_{3} n_{2}}^{\gamma \beta}\left(p_{2}\right)\right]^{*} q_{n_{3} n_{4}}^{\gamma \delta}\left(p_{3}\right)\left[q_{n_{1} n_{4}}^{\alpha \delta}\left(p_{4}\right)\right]^{*} \cdot \delta\left(p_{1}+p_{2}+p_{3}+p_{4}\right) \times \\
& \times\left\{\left(p_{1}-p_{2}\right) \cdot\left(p_{3}-p_{4}\right)+\left(p_{1}-p_{4}\right) \cdot\left(p_{3}-p_{2}\right)+\kappa^{2} z_{0}\left(n_{12}+n_{34}\right)+2 h_{0}^{2}\right\}
\end{aligned}
$$

The fourth and third order part of the interaction term are given by

$$
S_{\text {int }}^{(4)}=\frac{\pi}{4 \beta} z_{0} c_{0} \sum_{\alpha}\left\{\operatorname{tr}\left(\mathrm{I}_{0}^{\alpha}\left[q, q^{\dagger}\right]\right)\right\}^{2}+\frac{\pi}{2 \beta} z_{0} c_{0} \sum_{\alpha, n>0} \operatorname{tr}\left(\mathrm{I}_{n}^{\alpha}\left[q, q^{\dagger}\right]\right) \operatorname{tr}\left(\mathrm{I}_{-n}^{\alpha}\left[q, q^{\dagger}\right]\right)
$$

and

$$
S_{\text {int }}^{(3)}=-\frac{\pi}{\beta} z_{0} c_{0} \sum_{\alpha, n>0}\left\{\operatorname{tr} \mathrm{I}_{n}^{\alpha} q^{\dagger} \operatorname{tr}\left(\mathrm{I}_{-n}^{\alpha}\left[q, q^{\dagger}\right]\right)+\operatorname{tr}\left(\mathrm{I}_{n}^{\alpha}\left[q, q^{\dagger}\right]\right) \operatorname{tr} \mathrm{I}_{-n}^{\alpha} q\right\}
$$

Performing the contractions in (4.12) is straightforward but laborious. In the end we get an expression for $\mathcal{F}_{2}$ in terms of the basic propagators $D$ and $D^{c}$ 


$$
\begin{aligned}
\mathcal{F}_{2}= & \frac{1}{\sigma_{0}} \kappa^{2} z_{0} c_{0} \sum_{s>0} s \cdot \int_{p, q} D_{q}(s) D_{p}(s) \\
& +\frac{2}{\sigma_{0}}\left(\kappa^{2} z_{0} c_{0}\right)^{2} \sum_{m, n>0} \min (m, n) \int_{p, q}\left\{-D_{p}(n) D_{p}^{c}(n) D_{q}(m+n)\right. \\
& +D_{q}^{c}(m) D_{p}(n) D_{p}^{c}(n) \\
& -D_{q}^{c}(m) D_{p}^{c}(n) D_{\mathbf{p}+\mathbf{q}}(m+n) \\
& \left.-\kappa^{2} z_{0} c_{0} \cdot m \cdot D_{q}^{c}(m) D_{p}(n) D_{p}^{c}(n) D_{\mathbf{p}+\mathbf{q}}(m+n)\right\} .
\end{aligned}
$$

The overall factor $\min (n, m)$ in front of all the terms with more than two propagators can be roughly understood as follows. The summation $m>0$ always enters the stage when a redefinition occurs of the summation variable $n_{2}$ on $q_{n_{1} n_{2}}$ to a new summation variable $m=n_{1}-n_{2}>0$. This shift leaves behind a constraint on the range of $n_{1}$, namely $n_{1}<m$. The tracing with $\mathrm{I}_{n}$ in (4.14) and (4.15) induces a shift and a constraint of the type $n_{1}<n$ (or $n_{1}+n \geq m$ or something similar). In every contribution a free sum over $n_{1}$ finally occurs, yielding $\min (n, m)$ due to the constraints imposed on the summation interval.

We wish to present the result of (4.16) in the form (4.6, 4.7), i.e. as a frequency sum of corrections to $z_{0}$. This means we have to perform two momentum integrals and one frequency sum, while keeping one frequency fixed. This can be done in the following way:

$$
\begin{aligned}
\sum_{m, n>0} \min (m, n) \cdot f(m, n) & =\sum_{m=1}^{\infty} m \sum_{n=m+1}^{\infty} f(m, n)+\sum_{n=1}^{\infty} n \sum_{m=n+1}^{\infty} f(m, n) \\
& =\sum_{s=1}^{\infty} s\left\{\sum_{l=s+1}^{\infty} f(s, l)+\sum_{l=s+1}^{\infty} f(l, s)\right\} .
\end{aligned}
$$

Thus (4.16) can be rewritten in the form

$$
\begin{aligned}
\mathcal{F}_{2}= & \frac{1}{\sigma_{0}} \kappa^{2} z_{0} c_{0} \sum_{s>0} s \cdot \int_{p, q} D_{q}(s) D_{p}(s) \\
& +\frac{2}{\sigma_{0}}\left(\kappa^{2} z_{0} c_{0}\right)^{2} \sum_{s>0} \sum_{l>s} \int_{p, q} *\left\{-D_{q}(s) D_{q}^{c}(s) D_{p}(s+l)\right. \\
& -D_{q}(s+l) D_{p}(l) D_{p}^{c}(l) \\
& +D_{q}^{c}(s) D_{p}(l) D_{p}^{c}(l) \\
& +D_{q}(s) D_{q}^{c}(s) D_{p}^{c}(l) \\
& -2 D_{q}^{c}(s) D_{p}^{c}(l) D_{\mathbf{p}+\mathbf{q}}(s+l) \\
& -\kappa^{2} z_{0} c_{0} \cdot D_{q}(s) D_{q}^{c}(s) \cdot l \cdot D_{p}^{c}(l) D_{\mathbf{p}+\mathbf{q}}(s+l) \\
& \left.-\kappa^{2} z_{0} c_{0} \cdot s \cdot D_{q}^{c}(s) D_{p}(l) D_{p}^{c}(l) D_{\mathbf{p}+\mathbf{q}}(s+l)\right\}
\end{aligned}
$$

Calculations are now straightforward but cumbersome. Some details are presented in the Appendix. The final result for the poles in $\varepsilon$ in two loop contribution is given by

$$
\begin{aligned}
\mathcal{F}_{2}= & \frac{2}{\sigma_{0}} \kappa^{2} z_{0} \sum_{s>0} s \cdot h_{0}^{4 \varepsilon} \Omega_{d}^{2}\left\{-\frac{\left(1+h_{s}^{2} / h_{0}^{2}\right)^{\varepsilon}-1}{\varepsilon \cdot h_{s}^{2} / h_{0}^{2}} \cdot \frac{2+\ln \alpha}{\varepsilon}-\frac{\ln \left(1+h_{s}^{2} / h_{0}^{2}\right)}{\varepsilon}\right. \\
& \left.+\frac{1}{2 \varepsilon^{2}}+\frac{\pi^{2}}{2 \varepsilon}+\mathcal{O}\left(\varepsilon^{0}\right)\right\}
\end{aligned}
$$


where we have introduced $h_{s}^{2}=\kappa^{2} z_{0} s$ and put $\alpha=0$ whenever possible. It should be mentioned that in obtaining (4.25) drastic cancelations of many singular terms in $\alpha$ take place (see Appendix). Combining (4.7) and (4.25), one gets

$$
\mathcal{F}_{1}+\mathcal{F}_{2}=\Omega_{d} \cdot \frac{1}{\varepsilon} \frac{\left(h^{\prime 2}+{h_{s}^{\prime}}^{2}\right)^{1+\varepsilon}-\left({h^{\prime}}^{2}\right)^{1+\varepsilon}}{1+\varepsilon}+\frac{1}{\sigma^{\prime}} \sum_{s>0}{h_{s}^{\prime}}^{2} \cdot h_{0}^{4 \varepsilon} \Omega_{d}^{2}\left\{\frac{1}{\varepsilon^{2}}+\frac{\pi^{2}}{\varepsilon}\right\},
$$

where we make use of the one-loop renormalization of $h_{0}, \sigma_{0}$ and $z_{0}$ :

$$
\begin{aligned}
& t_{0} \rightarrow t^{\prime}=t_{0} \cdot\left\{1-\frac{h_{0}^{2 \varepsilon}}{\varepsilon} t_{0}\right\} \\
& z_{0} \rightarrow z^{\prime}=z_{0} \cdot\left\{1+\frac{h_{0}^{2 \varepsilon}}{2 \varepsilon} t_{0}\right\} \\
& h_{0}^{2} \rightarrow h^{\prime 2}=h_{0}^{2} \cdot\left\{1-[2+\ln \alpha] \frac{h_{0}^{2 \varepsilon}}{\varepsilon} t_{0}\right\} .
\end{aligned}
$$

Equations (4.27)-(4.29) imply the following renormalization of the quantity $h_{s}^{2} \propto t_{0} z_{0}$

$$
h_{s}^{2} \rightarrow{h_{s}^{\prime 2}}^{2} h_{s}^{2} \cdot\left\{1-\frac{h_{0}^{2 \varepsilon}}{2 \varepsilon} t_{0}\right\}
$$

Let us also present the result for the case when $h_{0}^{2}=0$, so that frequency serves as infrared regulator:

$$
\begin{aligned}
& \mathcal{F}_{0}=\frac{2 \Omega_{D}}{t_{0}} \sum_{s>0}^{\prime} h_{s}^{2} \\
& \mathcal{F}_{1}=\frac{2 \Omega_{D}}{t_{0}} \sum_{s>0}^{\prime} h_{s}^{2} \cdot \frac{h_{s}^{2 \varepsilon}}{\varepsilon(1+\varepsilon)} \frac{t_{0}}{2} \\
& \mathcal{F}_{2}=-\frac{2 \Omega_{D}}{t_{0}} \sum_{s>0}^{\prime} h_{s}^{2} \cdot \frac{h_{s}^{2 \varepsilon}}{\varepsilon^{2}} \frac{t_{0}^{2}}{8}\left[1-\varepsilon\left(\frac{\pi^{2}}{3}+4\right)+\mathcal{O}\left(\varepsilon^{2}\right)\right] .
\end{aligned}
$$

\section{SCALING RESULTS}

Our renormalization group program of the Finkelstein theory consisted of two separate parts. First we studied the general background field procedure in dimensional regularization and we focused primarily on how the interaction term affects the ultraviolet behaviour of the theory. This procedure provides two renormalization constants, $Z_{1}$ and $Z_{2}$. (From now on we consider the Coulomb case $\alpha=0$.)

The background field procedure and other, arbitrary, renormalization group program 14 do not provide us, however, with the full temperature and frequency dependence of physical observables such as the conductivity. This was the subject of the second part in which we recognized that observables should be constructed out of only those correlations or 'background' fields that leave the interaction term $S_{\mathrm{F}}$ invariant. There are two classes of such 
correlations: the free energy itself and the one denoted as 'linear response', which provide us with equilibrium statistical mechanics and transport theory, respectively. For these quantities we can derive general scaling results from the renormalization group.

First we summarize the results for the conductivity $\sigma_{0}$ and the free energy $\mathcal{F}_{0}+\mathcal{F}_{1}+\mathcal{F}_{2}$ as follows

$$
\begin{aligned}
\sigma^{\prime}(s) & =\frac{4 \Omega_{D}}{t_{0}} R_{\sigma}\left(t_{0}, h_{s}\right) \\
R_{\sigma} & =1+\frac{h_{s}^{2 \varepsilon}}{\varepsilon} t_{0}\left(1-\varepsilon\left[\frac{5}{2}-2 \ln 2\right]\right) .
\end{aligned}
$$

The $\ln T$-derivative of the free energy can be written as

$$
\frac{\partial \mathcal{F}}{\partial \ln T}=2 \Omega_{D} \sum_{s>0}\left(h_{s}^{\prime}\right)^{2} / t^{\prime}=2 \Omega_{D} \sum_{s>0} \frac{h_{s}^{2}}{t_{0}} M\left(t_{0}, h_{s}\right)
$$

where

$$
M\left(t_{0}, h_{s}\right)=1+\frac{h_{s}^{2 \varepsilon}}{2 \varepsilon} t_{0}+\frac{h_{s}^{4 \varepsilon}}{\varepsilon^{2}} t_{0}^{2}\left(-\frac{1}{8}+\varepsilon \frac{1}{4}\left[1+\frac{\pi^{2}}{6}\right]\right)
$$

These results are completely analogous to what has been obtained for the Heisenberg ferromagnet11. The quantity $M$ is the analogue of the magnetization in ferromagnetic language and we shall next follow up on the analysis of 11 . Equation (5.3) can be understood in terms of the effective parameters $h_{s}^{\prime}$ and $t^{\prime}$ with the latter defined by

$$
\frac{1}{t^{\prime}}=\frac{1}{t_{0}} R\left(t_{0}, h_{s}\right) \quad ; \quad R\left(t_{0}, h_{s}\right)=1+\frac{h_{s}^{2 \varepsilon}}{\varepsilon} t_{0}+\mathcal{O}\left(t_{0}^{2}\right) .
$$

Notice that the expressions for $\sigma^{\prime}$ and $1 / t^{\prime}$ in (5.1) and (5.4) are not necessarily the same, since they describe different physics (linear response and equilibrium statistical mechanics respectively). Nevertheless, both (5.1,5.3) and (5.4) can be used to extract the renormalization constants $Z_{1}$ and $Z_{2}$ obtained by putting

$$
t_{0}=\mu^{-2 \varepsilon} t Z_{1} \quad ; \quad \Omega_{D} \frac{h_{s}^{2}}{t_{0}}=\omega_{s} z_{0}=\omega_{s} \mu^{2 \varepsilon} z Z_{2}
$$

where $t, z$ now stand for the parameters of the renormalized theory. Following the scheme of minimal subtraction we get

$$
\begin{aligned}
& Z_{1}=1+t / \varepsilon \\
& Z_{2}=1-\frac{t}{2 \varepsilon}-\frac{t^{2}}{\varepsilon^{2}}\left(\frac{1}{8}+\varepsilon\left[\frac{\pi^{2}}{24}+\frac{3}{4}\right]\right) .
\end{aligned}
$$

The renormalization $\beta$ - and $\gamma$-functions are obtained as usual

$$
\beta=\frac{2 \varepsilon t}{1+t \frac{d \ln Z_{1}}{d t}} \quad ; \quad \gamma=\beta \frac{d \ln Z_{2}}{d t}
$$

yielding

$$
\beta=2 \varepsilon t-2 t^{2} \quad ; \quad \gamma=-t-t^{2}\left(3+\pi^{2} / 6\right) .
$$

Next we express the free energy and conductivity in terms of the renormalized parameters 


$$
\frac{\partial \mathcal{F}}{\partial \ln T}=2 \sum_{s>0} \mu^{2 \varepsilon} z \omega_{s} M \quad \frac{1}{t^{\prime}}=\mu^{2 \varepsilon} \frac{1}{t} R \quad \sigma^{\prime}=\mu^{2 \varepsilon} \frac{4 \Omega_{D}}{t} R_{\sigma}
$$

where $M, R$ and $R_{\sigma}$ can be written in scaling form following the method of characteristics

$$
\begin{aligned}
M & =M_{0}(t) g\left(\omega_{s} z \xi^{D} M_{0}\right) \\
R & =R_{0}(t) h\left(\omega_{s} z \xi^{D} M_{0}\right) \\
R_{\sigma} & =R_{0}(t) f\left(\omega_{s} z \xi^{D} M_{0}\right)
\end{aligned}
$$

with $M_{0}, R_{0}$ and $\xi$ determined from the renormalization group functions $\beta$ and $\gamma$ according to

$$
\begin{aligned}
& {\left[\mu \partial_{\mu}+\beta \partial_{t}\right] \xi(t)=0} \\
& {\left[\beta \partial_{t}-\gamma\right] M_{0}(t)=0} \\
& {\left[\beta \partial_{t}+2 \varepsilon-t^{-1} \beta\right] R_{0}(t)=0 .}
\end{aligned}
$$

More explicitly, in $2+2 \varepsilon$ dimensions we have for the metallic phase $\left(t<t_{c}\right)$

$$
\begin{aligned}
\xi & =\mu^{-1} t^{1 / 2 \varepsilon}\left(1-t / t_{c}\right)^{-\nu} \\
R_{0} & =\left(1-t / t_{c}\right)^{2 \varepsilon \nu} \\
M_{0} & =\left(1-t / t_{c}\right)^{\beta_{0}}
\end{aligned}
$$

where $t_{c}=\varepsilon+\mathcal{O}\left(\varepsilon^{2}\right)$ is the critical point $\left(\beta\left(t_{c}\right)=0\right)$ whereas

$$
\nu=-1 / \beta^{\prime}\left(t_{c}\right) \quad ; \quad \beta_{0}=-\nu \gamma\left(t_{c}\right) .
$$

It is interesting to conclude that the interacting electron gas is much closer to the physics of the Heisenberg ferromagnet than the free electron problem which has $\beta_{0}=0$, indicating that the density of states is nonsingular. The metal/insulator transition for interacting electrons is characterized by the appearance of a real order parameter. We will show below (section VI) that $\beta_{0} \neq 0$ in this case means that the specific heat becomes singular as one approaches from the metallic side.

Important conceptual quantities of the theory are the various length scales $\left(L_{\varphi}\right)$ induced by the phase breaking parameters such as temperature $T$, frequency $\omega$ and the parameter denoted as $h$. From the renormalization group we have

$$
\begin{aligned}
L_{\varphi}^{-D}\left(h_{0}\right) & =\left(h^{\prime}\right)^{2} \\
L_{\varphi}^{-D}(T) & =T z M_{0} g_{T}\left(T z \xi^{D} M_{0}\right) \\
L_{\varphi}^{-D}(\omega) & =\omega z M_{0} g_{\omega}\left(\omega z \xi^{D} M_{0}\right) .
\end{aligned}
$$

Finally, we can obtain 'equations of state' for the quantities $M$ and $R$. As shown in 11 to lowest order in $\varepsilon$, these quantities obey

$$
\begin{aligned}
& \frac{\omega_{s} z t}{M^{\delta}}=\left(t_{c} / t\right)^{1 / \varepsilon}\left(1-2 \varepsilon \nu \frac{1-t / t_{c}}{M^{1 / \beta_{0}}}\right)^{1 / \varepsilon} \\
& \frac{\omega_{s} z t}{R^{\kappa}}=\left(t_{c} / t\right)^{1 / \varepsilon}\left(1-\frac{1-t / t_{c}}{R^{1 / 2 \varepsilon \nu}}\right)^{1 / \varepsilon}
\end{aligned}
$$


with the following relations between the exponents

$$
D \nu=\beta_{0}(\delta+1) \quad ; \quad \kappa=\beta_{0} \delta /(2 \varepsilon \nu) .
$$

The equation of state for the conductivity is obtained by replacing $R \rightarrow R_{\sigma}$ and $\omega_{s} \rightarrow i \omega$ in (5.16).

\section{SPECIFIC HEAT}

In order to make contact with an important quantity like the specific heat, we will first evaluate the derivative $\partial \mathcal{F} / \partial \ln T$ from the fermionic path integral for the case of free particles, and compare the results to those obtained from $Q$-field theory. By the rules of statistical mechanics we have

$$
-\frac{\partial \ln Z}{\partial \ln \beta}=N_{r} \beta(\bar{E}-\mu \bar{N})
$$

where $\bar{E}$ is the average energy and $\bar{N}$ the average number of electrons. Since the specific heat is defined as the change of $\bar{E}$ with varying $T$ for a fixed number of particles, we still need some procedure that eliminates the explicit $\mu$-dependence in (6.1)

\section{A. Free particles}

From the original fermionic path integral we obtain directly

$$
V_{D} \frac{\partial \mathcal{F}}{\partial \ln \beta}=\sum_{n} i \omega_{n} \operatorname{Tr} \frac{1}{i \omega_{n}+\mu-H_{0}}
$$

with $\ln Z=-V_{D} N_{r} \mathcal{F}$. The 'Tr' stands for the trace over position space and $V_{D}$ is the volume

of the system. By writing the frequency sum as a contour integral in the usual manner,

$$
V_{D} \frac{\partial \mathcal{F}}{\partial \ln \beta}=-\frac{\beta}{2 \pi i} \oint d z \frac{z}{e^{\beta z}+1} \operatorname{Tr} \frac{1}{z+\mu-H_{0}},
$$

we obtain the more transparent expression

$$
\beta V_{D} \int_{-\infty}^{\infty} d \epsilon \frac{\epsilon}{e^{\beta \epsilon}+1} \rho(\epsilon)
$$

where $\rho(\epsilon)$ is the density of states at energy $\mu+\epsilon$ and $V_{D}$ the volume of the system. The equivalence between Eq (6.1) and (6.4) is now easily established. By splitting Eq (6.4) into zero- $T$ and finite- $T$ parts we finally obtain

$$
\begin{aligned}
\frac{\partial \mathcal{F}}{\partial \ln \beta} & =\beta\left(f_{0}+f_{T}\right) \\
& =\beta \int_{-\infty}^{0} d \epsilon \epsilon \rho(\epsilon)+\beta \int_{0}^{\infty} d \epsilon \frac{\epsilon}{e^{\beta \epsilon}+1}[\rho(\epsilon)+\rho(-\epsilon)] \\
& =\beta\left(-\int_{-\infty}^{\mu} d E n(E)+\int_{0}^{\infty} d \epsilon \frac{\epsilon}{e^{\beta \epsilon}+1}[\rho(\epsilon)+\rho(-\epsilon)]\right)
\end{aligned}
$$


with $n(E)$ the total number of states with energy less than $E(\partial n(E) / \partial E$ is equal to the density of states). The $f_{T}$ determines the well known specific heat of the free electron gas for low temperatures $\left(c_{v}=\partial f_{T} / \partial T=\gamma T\right)$. Next, we wish to redo the various steps which take us from $\mathrm{Eq}(6.2)$ to (6.5), but now starting from the effective theory in $Q$. We obtain, instead of $\mathrm{Eq}$ (6.2)

$$
\frac{\partial \mathcal{F}}{\partial \ln \beta}=\pi \rho_{0} N_{r}^{-1} \operatorname{tr}\langle\omega Q\rangle=2 \pi \rho_{0} \sum_{n \geq 0}^{\prime} \omega_{n}
$$

where $\omega_{n}=\pi(2 n+1) / \beta$ and the prime on the summation indicates that the sum involves a cutoff. By taking a simple exponential form, i.e. replacing Eq (6.6) by

$$
2 \pi \rho_{0} \sum_{n \geq 0} \omega_{n} e^{-\omega_{n} \tau_{0}}
$$

and by redoing the various steps which take us from Eq (6.2) to (6.5), one is easily convinced that the low $T$ behaviour of the specific heat is identically the same as was obtained before. To be more specific, Eq. (6.7) gives

$$
f_{0}=\int_{0}^{\infty} d \varepsilon \varepsilon \rho_{0} e^{-\varepsilon \tau_{0}} \quad \text { and } \quad f_{T}=\int_{0}^{\infty} d \varepsilon \frac{\varepsilon}{e^{\beta \varepsilon}+1} 2 \rho_{0} .
$$

(All the dependence on the cutoff $\tau_{0}$ is absorbed in the zero- $T$ part of Eq (6.5), i.e. $f_{0}$, and this quantity is of secondary interest). Hence, the low- $T$ specific heat of the electron gas is correctly retained by the effective $Q$-field formalism.

\section{B. Coulomb interactions; quasiparticles}

Next we embark on the Coulomb interaction problem. We obtain from the Finkelstein action, instead of Eq (6.6),

$$
\frac{\partial \mathcal{F}}{\partial \ln \beta}=\frac{\pi}{2 \beta} z_{0}\left\langle\sum_{n}^{\prime} \operatorname{tr}\left[\mathrm{I}_{n}^{\alpha}, Q\right]\left[\mathrm{I}_{-n}^{\alpha}, Q\right]\right\rangle .
$$

Besides the renormalizable quantity in the $Q$-matrix fields, there are also other, $Q$ independent, contributions to (6.9) that are still left over from the underlying theory of longitudinal modes (see [I]). These contributions, however, are of a Fermi liquid type and, hence, of secondary interest. Using the results of Section $\square$, we write (6.9) as follows

$$
-2 z_{0} \sum_{n>0} \omega_{n} M\left(\omega_{n}\right) e^{-\omega_{n} \tau_{0}}
$$

where the sum is now over bosonic Matsubara frequencies $\omega_{n}=2 \pi n / \beta$ and, as before, we introduced an arbitrary $\tau_{0}$ for convergence purposes. Notice that the quantity $M\left(\omega_{n}\right)$, in contrast to the density of states $\rho_{0}$ in the expression for the free electron problem (6.7), now acquires nontrivial $\omega_{n}$-dependence. We proceed by evaluating the discrete sum in (6.10) as a contour integral. Repeating the same steps which lead to (6.5) we obtain

$$
\frac{\partial \mathcal{F}}{\partial \ln \beta}=\beta\left(f_{0}+f_{T}\right)
$$


where

$$
f_{0}=-\frac{z_{0}}{\pi} \int_{0}^{\infty} d \epsilon \epsilon M(\epsilon) e^{-\epsilon \tau_{0}}
$$

and $f_{T}$ can be written as

$$
f_{T}=\frac{z_{0}}{\pi} \int_{0}^{\infty} d \epsilon \frac{\epsilon}{e^{\beta \epsilon}-1} \rho_{q p}(\epsilon)
$$

with

$$
\rho_{q p}(\epsilon)=e^{i \epsilon \tau_{0}} M(-i \epsilon)+e^{-i \epsilon \tau_{0}} M(i \epsilon) .
$$

As before (Eq 6.5), the specific heat is obtained as $c_{V}=\partial f_{T} / \partial T$. The quantity $\rho_{q p}(\epsilon)$ is now identified as the quasiparticle density of states of the interacting system.

The expression for $f_{T}, \mathrm{Eq}$ (6.13), is the most important result of this section and we make use of the results for $M\left(\omega_{n}\right)$, section $\nabla$, in order to extract the behavior of the specific heat. First, in the metallic phase in $2+2 \varepsilon$ dimensions, the arbitrary cutoff factor containing $\tau_{0}$ in $f_{T}$ does not contribute to the leading behaviour of $c_{V}$ at low $T$, and $\tau_{0}$ can be safely put to zero. This can be seen most simply from the behaviour near the fixed points at $t=0$,

$$
\begin{aligned}
t & \rightarrow 0 & & \text { (Fermi liquid phase) } \\
M\left(\omega_{n}\right) \approx 1 & & \rho_{q p} \rightarrow 2 \cos \left(\epsilon \tau_{0}\right) \approx 2 & c_{v} \rightarrow \gamma_{0} T
\end{aligned}
$$

and at $t=t_{c}$, in which case we have

$$
\begin{aligned}
t=t_{c} & \text { (critical phase) } \\
M\left(\omega_{n}\right) \approx\left|\omega_{n}\right|^{1 / \delta} & \rho_{q p} \approx|\epsilon|^{1 / \delta} 2 \cos \left(\frac{\pi}{2 \delta}+\epsilon \tau_{0}\right) \quad c_{v} \rightarrow \gamma^{*} T^{1+1 / \delta} .
\end{aligned}
$$

Hence, approaching the metal-insulator transition from the metallic side, the (bosonic) quasiparticle density of states develops a (Coulomb) gap and the exponent $1 / \delta$ is of order $\varepsilon=(D-2) / 2$. More generally, we have the following scaling result,

$$
\rho_{q p}(\epsilon)=M_{0} G\left(\epsilon z \xi^{D} M_{0}\right)=M(-i \epsilon)+M(i \epsilon)
$$

which may be obtained in closed form following the results for $M$ of section $\square$.

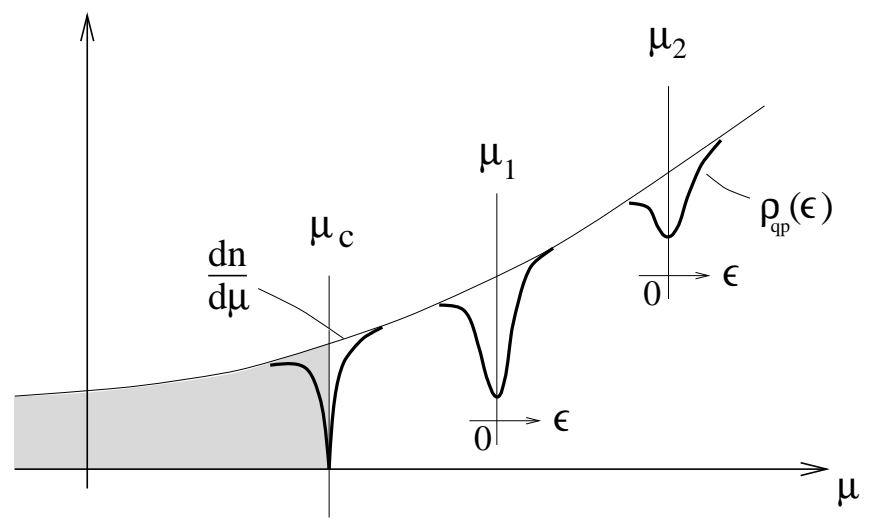

FIG. 4. The quasiparticle density of states in $2+2 \varepsilon$ dimensions for different values of the chemical potential $\left(\mu_{1}, \mu_{2}\right.$ and $\left.\mu_{c}\right)$. The critical value $\mu_{c}$ separates the insulating phase (shaded region) from the metallic phase (unshaded region); see text. 
For completeness we give a sketch of $\rho_{q p}(\epsilon)$ as compared to the thermodynamic density of states $\partial n / \partial \mu$, Fig. 4 . The $\partial n / \partial \mu$ does not enter the effective $Q$-theory and is taken as a regular function of the chemical potential, since it is solely determined by the underlying theory with the $P$ matrix field variables. Figure 1 indicates that $\rho_{q p}(\epsilon)$ as a whole moves along as one varies the chemical potential $\mu$ with $\epsilon$ really standing for the thermal energy fluctuations for a given, fixed $\mu$. The effective band width $(\Delta \epsilon)$ for quasiparticle excitation is on the order of $\hbar / \tau_{0}$, i.e. the characteristic energy scale below which the Coulomb effects become noticeable. It is, of course, understood that for free electrons there is no distinction between $\rho(\epsilon)(6.5)$ and $\partial n / \partial \mu$, and the quasiparticles are the electrons themselves.

The formation of a gap in $\rho_{\mathrm{qp}}$ is reminiscent of the Efros-Shklovskii Coulomb gap 11 in the tunneling density of states in the localized phase. However, since the tunneling density of states is not a gauge invariant object $\mathrm{\theta}$, the relation between the two is not obvious. In order to make contact with the heuristic approach by Efros and Shklovskilit, it is necessary to encompass the limitations of the perturbative renormalization group and find a way to penetrate into the regime of strong coupling. We elaborate further on this point in Section D.

\section{Crossing over between free electrons and quasiparticles}

Let us next come back to our earlier discussion (section ПIA), where we replaced the original problem with Coulomb interactions (1.4) by a simpler one (2.1) introducing the parameter $c_{0}$ (or $\alpha$ ). We argued that since the Coulomb term $S_{\mathrm{U}}$ is irrelevant we obtain the same results by putting $c_{0}=1(\alpha=0)$ in the simpler theory. We are now in a position to appreciate the fact that the two theories really stand for entirely different physical scenarios by which the free electron and Coulomb theories are related.

As already mentioned before, the parameter $\alpha$ in the simple theory can (loosely) be interpreted in terms of the 'range' of the electron-electron interactions, and the results indicate that infinite range interactions $(\alpha=0)$ and finite range interactions $(\alpha>0)$ belong to different universality classes. On the other hand, the Coulomb term $S_{\mathrm{U}}$ in the original theory really interpolates between the action for free particles at high momenta and the Finkelstein theory which only appears in the limit of large distances (relative to the screening length).

This means that the true, physical theory predicts free electron behaviour with FermiDirac statistics at high $T$ (6.5) and quasiparticle behaviour with scaling and Bose-Einstein statistics at low $T$ (6.15). This crossover mechanism for which the Coulomb term $S_{\mathrm{U}}$ is responsible obviously applies to other physical quantities such as $\sigma_{x x}$. This mechanism is extremely important, since it has been experimentally shown to apply to the more complicated metallic phases of the quantum Hall regime as well. More specifically, the transport data taken from low mobility heterostructures follow the well known free electron behaviour at high $T(4 \mathrm{~K} \leq T<20 \mathrm{~K})$,

$$
\sigma_{i j}(T)=\int d E \frac{\partial f(T)}{\partial E} \sigma_{i j}^{0}(E)
$$

Here $f(T)$ stands for the Fermi-Dirac distribution (see 6.2) and $\sigma_{i j}^{0}$ are the mean field conductances with varying energy $E$.

This behaviour crosses over smoothly but rapidly into a scaling behaviour in $T$, which was observed at lower temperatures $(20 \mathrm{mK} \leq T<4 \mathrm{~K})$ only. This, then, indicates that the 
complete theory as given by (1.1) has all the ingredients necessary to describe the complex phenomenon of plateau transitions in the quantum Hall regime.

\section{Strong coupling aspects}

The analogy with the Heisenberg ferromagnet as discussed in previous sections naturally supposes that one can take the theory one step further by extending the ferromagnetic language to include the insulating phase as well. For example, for ordinary ferromagnets we know that, since there is no phase transition in the symmetric phase, the magnetization $M\left(\omega_{n}\right)$ should become a regular and odd function of the 'external field' $\omega_{n}$. From the renormalization group it can be shown that such a condition on $M\left(\omega_{n}\right)$ implies that the $R\left(\omega_{n}\right)$ (but not $R_{\sigma}$ !) is regular as well but it must be an even function of $\omega_{n}$. This analyticity statement (Griffith analyticity) would imply in our case, however, that the quasiparticle density of states collapses, i.e. (6.16) vanishes to all orders in $\epsilon$ !

This oddly looking conclusion actually has an extremely important physical significance and it can easily be understood on the basis of our discussion of $\mathcal{F}$-invariance. First we remark that a vanishing $\rho_{q p}(\epsilon)$ is a direct consequence of working with an action where the Coulomb part $S_{\mathrm{U}}$ has been replaced by a symmetry braking term $S_{\alpha}$ and where the 'external' symmetry breaking $\alpha$ has been put equal to zero. Notice that for Fermi level quantities like $\rho_{q p}(\epsilon)$ and $\sigma(\omega)$ the role of $\alpha$ itself is analogous to the role of the magnetic field in the Heisenberg ferromagnet.

This is evident from the linear response procedure, where the perturbing vector potential can be considered as a 'generator' of $\mathcal{F}$-symmetry which is broken by the external $\alpha$-field. Hence we should expect that the quantities $\rho_{q p}$ and $\sigma$, which are a direct measure of the low energy excitations of the electron gas, become regular in $\alpha$ as one enters deeply into the insulating phase. In other words, they become zero by putting $\alpha$ equal to zero.

The important conclusion that can be drawn from this discussion is that the Coulomb term $S_{\mathrm{U}}$ (1.4) can no longer be regarded as 'irrelevant' but, instead, it is going to completely determine the physics of the insulating phase.

We conclude this section by giving a brief digression on the general significance of the Coulomb part of the action. This, then, serves as a starting point for a more extended renormalization group analysis which will be reported elsewhere. For this purpose, let us go back to the original theory (1.1) and compute the response to an external field $A_{\mu}$. At a tree level, the result is given by ( $\sec ^{5}$ )

$$
S[A]=-\sigma_{x x}^{0} \sum_{\alpha, n>0} \int \frac{d^{2} q}{(2 \pi)^{2}} n \overline{\left(z_{i}\right)_{n}^{\alpha}(q)}\left[\delta_{i j}-\frac{q_{i} q_{j}}{q^{2}+\kappa^{2} n U^{-1}(q)}\right]\left(z_{j}\right)_{n}^{\alpha}(q)
$$

where $\vec{z}_{n}^{\alpha}$ is defined as $\vec{A}_{n}^{\alpha}-i \nabla\left(A_{\tau}\right)_{n}^{\alpha} / \omega_{n}$. The result (6.18) can be used to show that the nature of particle transport in metals changes depending on what length scale one probes the system at. From the definition for the particle density $n$

$$
-\beta n_{m}^{\alpha}(q)=\frac{\delta S\left[A_{\mu}\right]}{\delta\left(A_{\tau}\right)_{-m}^{\alpha}(-q)}
$$


we obtain, following 6.18

$$
\left[\omega_{m}+\frac{1}{4} \sigma_{x x}^{0} q^{2} U(q)\right] n_{m}^{\alpha}(q)=i \vec{q} \cdot\left(\vec{\jmath}_{\mathrm{ext}}\right)_{m}^{\alpha}(q) .
$$

The $\vec{\jmath}_{\text {ext }}$ is the current density induced by external fields. We have obtained this current density by using $\vec{z}_{m}^{\alpha}=i \vec{E}_{m}^{\alpha} / \omega_{m}$ and $\vec{\jmath}_{\text {ext }}=\frac{\sigma_{x x}^{0}}{2 \pi} \vec{E}$. For large momenta we have

$$
U(q) \approx \frac{2}{\pi} \rho^{-1} \quad \text { as } \quad|q| \rightarrow \infty
$$

and $(6.20)$ becomes the expression for particle conservation in a diffusive system driven by an external potential,

$$
\partial_{t} n_{c}+\nabla \cdot\left(\vec{\jmath}_{\text {ext }}+\vec{\jmath}_{\text {diff }}\right)=0
$$

where $n_{c}=-n$ is the charge density, $\vec{\jmath}_{\text {diff }}=-D \nabla n_{c}$ with $D=\sigma_{x x}^{0} /(2 \pi \rho)$ the diffusion constant. On the other hand, in the small $q$ limit one has

$$
U(q) \rightarrow \frac{2}{\pi} U_{0}(q) \quad \text { as } \quad|q| \rightarrow 0 .
$$

We now write $\vec{E}_{c}(q)=-i \vec{q} U_{0}(q) n_{c}(q)$ or, in position space,

$$
\vec{E}_{c}(x)=-\nabla \int d^{2} x^{\prime} U_{0}\left(x, x^{\prime}\right) n_{c}\left(x^{\prime}\right)
$$

for the local electric field as a result of the interaction with all the other electrons in the system. We now have, instead of (6.22),

$$
\partial_{t} n_{c}+\nabla \cdot\left(\vec{\jmath}_{\mathrm{ext}}+\vec{\jmath}_{c}\right)=0
$$

where $\vec{\jmath}_{c}=\frac{\sigma_{x x}^{0}}{2 \pi} \vec{E}_{c}$.

Equation (6.25) indicates that 'detailed balance' no longer stands for mutually compensating electric and 'diffusive' currents. Rather, the external field is canceled by the internally generated electric field due to the Coulomb forces. The length scale at which one crosses over from the diffusion dominated regime (6.22) to the 'Coulomb driven' regime (6.25) is given by the Debye static screening length $(2 \pi \rho)^{-1}$.

Let us next come back to the subject of linear response. It is clear that for the computation of conductivities one generally has to take the limit $q \rightarrow 0$ first and then $\omega \rightarrow 0$. Notice that the abovementioned complications with with the limit $\alpha \rightarrow 0$ are, in fact, foreshadowed by the response at tree level (6.18). For instance, working in a theory where $U(q)$ is replaced by $\alpha^{-1}$ (as was done for renormalization group purposes) then linear response leads to the correct result provided $\alpha \rightarrow 0$ in the end. As one approaches the insulating phase, however, it seems from (6.18) that the renormalization group result $\left(\sigma \propto \alpha \xi^{2} \omega\right)$ is indeed the only way of obtaining a smooth $\alpha \rightarrow 0$ limit. This indicates that the theory with $\alpha=0$ develops an energy gap. However, in the presence of $U(q)$ it is more likely that $\sigma \propto \xi \omega$ in the insulating phase, which means that the dominant energy scale is now determined by the Coulomb potential. 


\section{CONCLUSION}

In this paper we have reported the results of a perturbative renormalization group analysis (to two loop order) of the Finkelstein theory of localization and interaction effects. This theory has fundamental significance for the quantum Hall effect and it is part of a unifying action as proposed in our previous work

We have shown that the infrared behaviour of the theory (i.e. the limit $h_{0} \rightarrow 0$ ) can only be extracted from a limited class of $(\mathcal{F}$-invariant $)$ correlation functions. This insight enabled us to identify several new quantities in the theory, such as the 'order parameter' of the metal-insulator transition in $2+2 \varepsilon$ dimensions and the 'Coulomb gap' which enters through the (bosonic) quasiparticle density of states into the expression for the specific heat.

$\mathcal{F}$-invariance has also fundamental consequences for the insulating phase, where ordinary perturbation theory is no longer valid. To this end, we exploited the analogy of the Finkelstein theory with the more familiar theory of the classical Heisenberg ferromagnet. We have shown that the transport problem in this case must be completely dominated by the Coulomb part of the action $\left(S_{\mathrm{U}}\right)$ which is usually discarded on the basis of naive scaling dimensions. The appropriate way of demonstrating this is by computing the anomalous dimension of the operators in $S_{U}$. This requires a more extended renormalization group program than the one presented in this paper. Progress in this direction, along with the possible consequences for the plateau transition in the quantum Hall regime, will be reported elsewhere.

\section{ACKNOWLEDGEMENTS}

This research was supported in part by INTAS (grant \#96-0580).

\section{Appendix}

In this appendix we present the final expressions for the various terms in (4.17)-(4.24) together with some calculational details. The calculation of (4.17)-(4.21) is straightforward because the internal momenta decouple, and we just present the results (only pole terms in $\varepsilon)$, taking the limit $\alpha \rightarrow 0$ wherever possible:

$$
\begin{aligned}
& \int_{p, q} D_{q}(s) D_{p}(s) \rightarrow \Omega_{D}^{2} z_{0} t_{0}^{2} \frac{h_{0}^{4 \varepsilon}}{\varepsilon^{2}}\left(1+\widehat{\omega}_{s}\right)^{2 \varepsilon}\left[\frac{\pi \varepsilon}{\sin \pi \varepsilon}\right]^{2} \\
& \kappa^{2} z_{0} \sum_{l>s} \int_{p, q} D_{q}(s) D_{q}^{c}(s) D_{p}(s+l) \rightarrow \Omega_{D}^{2} h_{0}^{4 \varepsilon} \cdot \frac{1}{\varepsilon^{2}(1+\varepsilon)}\left\{\left(1+2 \widehat{\omega}_{s}\right)^{1+\varepsilon} \frac{\left(1+\widehat{\omega}_{s}\right)^{\varepsilon}-1}{\widehat{\omega}_{s}}\right\} \\
& \kappa^{2} z_{0} \sum_{l>s} \int_{p, q} D_{q}(s+l) D_{p}(l) D_{p}^{c}(l) \rightarrow \Omega_{D}^{2} h_{0}^{4 \varepsilon} \cdot\left\{\frac{\mathrm{I}_{3}(\varepsilon)}{2 \varepsilon^{2}} \frac{\alpha^{-\varepsilon}-1}{\varepsilon}+\frac{1}{\varepsilon} \int_{0}^{1} \frac{d x}{x} \ln \left(1+x \widehat{\omega}_{s}\right)\right\} \\
& \kappa^{2} z_{0} \sum_{l>s} \int_{p, q} D_{q}^{c}(s) D_{p}(l) D_{p}^{c}(l) \rightarrow \Omega_{D}^{2} h_{0}^{4 \varepsilon} \cdot\left\{-\frac{\mathrm{I}_{3}(\varepsilon)}{\varepsilon^{2}} \ln \alpha \cdot \frac{[\Gamma(1-\varepsilon)]^{2}}{\Gamma(1-2 \varepsilon)}\right. \\
&\left.+\frac{1}{\varepsilon} \int_{0}^{1} \frac{d x}{x} \ln \left(1+x \widehat{\omega}_{s}\right)\right\}
\end{aligned}
$$




$$
\begin{aligned}
\kappa^{2} z_{0} \sum_{l>s} \int_{p, q} D_{q}(s) D_{q}^{c}(s) D_{p}^{c}(l) \rightarrow & \Omega_{D}^{2} h_{0}^{4 \varepsilon} \cdot\left\{\frac{1}{\alpha \varepsilon^{2}} \frac{(1+z)^{\varepsilon}-(1+\alpha z)^{\varepsilon}}{z(1-\alpha)(1+\varepsilon)}\right. \\
& \left.+\frac{1}{\varepsilon^{2}}\left[\left(1+\widehat{\omega}_{s}\right)^{\varepsilon}-1\right]\right\},
\end{aligned}
$$

where we have defined $\widehat{\omega}_{s}=h_{s}^{2} / h_{0}^{2}$ and $\mathrm{I}_{3}(\varepsilon)$ stands for the combination

$$
\mathrm{I}_{3}(\varepsilon)=\frac{\Gamma(1-2 \varepsilon)}{[\Gamma(1-\varepsilon)]^{2}}\left[\frac{\pi \varepsilon}{\sin \pi \varepsilon}\right]^{2} \approx 1+\frac{\pi^{2}}{2} \varepsilon^{2}+\mathcal{O}\left(\varepsilon^{3}\right) .
$$

The calculation of (4.22) is more difficult but standard. As far as we are interested only in poles in $\varepsilon$ we can replace the sum over $l$ by an integral and put the lower limit to zero rather that $\omega_{s}$ :

$$
\frac{2 \pi}{\beta} \sum_{l>s} \rightarrow \int_{0}^{\infty} d \omega_{l}+\mathcal{O}\left(\varepsilon^{0}\right)
$$

Using the Feynman trick one can write

$$
\begin{aligned}
& \kappa^{2} z_{0} \sum_{l>s} \int_{p, q} D_{q}^{c}(s) D_{p}^{c}(l) D_{\mathbf{p}+\mathbf{q}}(s+l)=4 \frac{z_{0}}{\sigma_{0}} \int_{0}^{\infty} d \omega_{l} \int_{p, q} \int_{0}^{1} \prod_{i=1}^{3} d x_{i} \times \\
& \times \frac{2 \cdot \delta\left(1-\sum_{i} x_{i}\right)}{\left[h_{0}^{2}+q^{2} x_{12}+p^{2} x_{23}+2 \mathbf{p q} x_{3}+\kappa^{2} s\left(\alpha x_{1}+x_{3}\right)+\kappa^{2} l\left(\alpha x_{2}+x_{3}\right)\right]^{3}},
\end{aligned}
$$

where $x_{13}=x_{1}+x_{2}$ and $x_{23}=x_{2}+x_{3}$. By shifting $p \rightarrow p-q x_{3} / x_{23}$ we can decouple $p$ and $q$ in the denominator, after which we are able to perform the integrals over $p, q$ and $\omega_{l}$, with the result

$$
-\frac{\mathrm{I}_{3}(\varepsilon)}{2 \varepsilon} \Omega_{D}^{2} h_{0}^{4 \varepsilon} \int_{0}^{1} \prod_{i=1}^{3} d x_{i} \frac{\delta\left(1-\sum_{i} x_{i}\right)}{\left[x_{1} x_{2}+x_{1} x_{3}+x_{2} x_{3}\right]^{1+\varepsilon}} \cdot \frac{\left[1+\widehat{\omega}_{s}\left(\alpha x_{1}+x_{3}\right)\right]^{2 \varepsilon}}{\left(\alpha x_{2}+x_{3}\right)} .
$$

Introducing new variables $x \in[0, \infty]$ and $u \in[0,1]$ as follows

$$
x_{1}=\frac{x}{x+1} \quad ; \quad x_{2}=\frac{u}{x+1} \quad ; \quad x_{3}=\frac{1-u}{x+1}
$$

formula $(\mathrm{A} 2)$ can be written in the form

$$
-\frac{\mathrm{I}_{3}(\varepsilon)}{2 \varepsilon} \Omega_{D}^{2} h_{0}^{4 \varepsilon} \int_{0}^{1} \frac{d u}{\alpha u+1-u} \int_{0}^{\infty} d x \frac{\left[1+\widehat{\omega}_{s}(1-u)+x\left(1+\alpha \widehat{\omega}_{s}\right)\right]^{2 \varepsilon}}{[x+u(1-u)]^{1+\varepsilon}} .
$$

Using a well-known integral representation for the hypergeometric function, the $x$-integral can be performed and we get the following expression for (A1)

$$
-\frac{\mathrm{I}_{3}(\varepsilon)}{2 \varepsilon} \Omega_{D}^{2} h_{0}^{4 \varepsilon}\left[\mathrm{I}_{1}-\frac{[\Gamma(1-\varepsilon)]^{2}}{\Gamma(1-2 \varepsilon)} \cdot \mathrm{I}_{2}\right]
$$

where 


$$
\begin{aligned}
& \mathrm{I}_{1}=\int_{0}^{1} d u \frac{[u(1-u)]^{-\varepsilon}}{\alpha u+1-u}\left[1+\widehat{\omega}_{s}(1-u)\right]^{2 \varepsilon}{ }_{2} \mathrm{~F}_{1}\left(1,-2 \varepsilon ; 1-\varepsilon ; \frac{u(1-u)\left(1+\alpha \widehat{\omega}_{s}\right)}{1+\widehat{\omega}_{s}(1-u)}\right) \\
& \mathrm{I}_{2}=2 \int_{0}^{1} \frac{d u}{\alpha u+1-u}\left\{1+(1-u)\left[\widehat{\omega}_{s}-u\left(1+\alpha \widehat{\omega}_{s}\right)\right]\right\}^{\varepsilon} .
\end{aligned}
$$

We are interested in the limit $\alpha \rightarrow 0$, which corresponds to the case of real electrons. But if one puts $\alpha=0$ in the above formulae then (A6) becomes divergent on the upper limit. The integral (A5) will be proportional to $\varepsilon^{-1}$ making the whole contribution (A1) proportional to $\varepsilon^{-3}$ and causing the renormalization group equation to become singular. For this reason we first rewrite (A5) in the following form

$$
\begin{aligned}
\mathrm{I}_{1}= & \int_{0}^{1} d u \frac{[u(1-u)]^{-\varepsilon}}{\alpha u+1-u} \\
& +\int_{0}^{1} d u \frac{[u(1-u)]^{-\varepsilon}}{\alpha u+1-u}\left\{\left[1+\widehat{\omega}_{s}(1-u)\right]^{2 \varepsilon}-1\right\} \\
& +\int_{0}^{1} d u \frac{[u(1-u)]^{-\varepsilon}}{\alpha u+1-u}\left[1+\widehat{\omega}_{s}(1-u)\right]^{2 \varepsilon} \times \\
& \times\left\{{ }_{2} \mathrm{~F}_{1}\left(1,-2 \varepsilon ; 1-\varepsilon ; \frac{u(1-u)\left(1+\alpha \widehat{\omega}_{s}\right)}{1+\widehat{\omega}_{s}(1-u)}\right)-1\right\} .
\end{aligned}
$$

The first integral (A7) can be done exactly while in the other two we can safely put $\alpha=0$, because their integrands behave smoothly when $u \rightarrow 1$ even for $\alpha=0$. Thus $\mathrm{I}_{1}$ can be written in the form

$$
\begin{aligned}
\mathrm{I}_{1}= & \frac{1}{\varepsilon}\left\{\alpha^{-\varepsilon} \frac{\pi \varepsilon}{\sin \pi \varepsilon}-\frac{[\Gamma(1-\varepsilon)]^{2}}{\Gamma(1-2 \varepsilon)}\right\}+\mathcal{O}\left(\alpha^{1-\varepsilon}\right) \\
& +\int_{0}^{1} d u \frac{[u(1-u)]^{-\varepsilon}}{1-u}\left\{\left[1+\widehat{\omega}_{s}(1-u)\right]^{2 \varepsilon}-1\right\} \\
& +\int_{0}^{1} d u \frac{[u(1-u)]^{-\varepsilon}}{1-u}\left[1+\widehat{\omega}_{s}(1-u)\right]^{2 \varepsilon}\left\{{ }_{2} \mathrm{~F}_{1}\left(1,-2 \varepsilon ; 1-\varepsilon ; \frac{u(1-u)}{1+\widehat{\omega}_{s}(1-u)}\right)-1\right\} .
\end{aligned}
$$

Making an $\varepsilon$-expansion and then using the following asymptotic formula for the hypergeometric ${ }_{2} \mathrm{~F}_{1}$ function, which follows directly from its definition,

$$
{ }_{2} \mathrm{~F}_{1}(1,-2 \varepsilon ; 1-\varepsilon ; z)=1+2 \varepsilon \ln (1-z)+\mathcal{O}\left(\varepsilon^{2}\right)
$$

we get the final answer for $\mathrm{I}_{1}$

$$
\begin{aligned}
\mathrm{I}_{1}= & \frac{1}{\varepsilon}\left\{\alpha^{-\varepsilon} \frac{\pi \varepsilon}{\sin \pi \varepsilon}-\frac{[\Gamma(1-\varepsilon)]^{2}}{\Gamma(1-2 \varepsilon)}\right\} \\
& +2 \varepsilon \int_{0}^{1} \frac{d u}{1-u} \ln \left[1+(1-u)\left(\widehat{\omega}_{s}-u\right)\right]+\mathcal{O}\left(\varepsilon^{2}\right) .
\end{aligned}
$$

The integral $\mathrm{I}_{2}$ (A6) can be treated in the same way 


$$
\begin{aligned}
\mathrm{I}_{2}= & 2 \int_{0}^{1} \frac{d u}{\alpha u+1-u}+2 \int_{0}^{1} \frac{d u}{1-u}\left\{\left[1+(1-u)\left(\widehat{\omega}_{s}-u\right)\right]^{\varepsilon}-1\right\} \\
= & -2 \ln \alpha+\mathcal{O}(\alpha \ln \alpha) \\
& +2 \varepsilon \int_{0}^{1} \frac{d u}{1-u} \ln \left[1+(1-u)\left(\widehat{\omega}_{s}-u\right)\right]+\mathcal{O}\left(\varepsilon^{2}\right) .
\end{aligned}
$$

Substitution of (A10) and (A11) into (A3) gives the final answer for the pole terms in (A1)

$$
\begin{aligned}
& \kappa^{2} z_{0} \sum_{l>s} \int_{p, q} D_{q}^{c}(s) D_{p}^{c}(l) D_{\mathbf{p}+\mathbf{q}}(s+l) \\
& =\Omega_{D}^{2} h_{0}^{4 \varepsilon} \cdot \frac{\mathrm{I}_{3}(\varepsilon)}{2 \varepsilon}\left\{-\frac{2 \ln \alpha}{\varepsilon} \frac{[\Gamma(1-\varepsilon)]^{2}}{\Gamma(1-2 \varepsilon)}-\frac{1}{\varepsilon} \frac{\alpha^{-\varepsilon}-1}{\varepsilon} \cdot \frac{\pi \varepsilon}{\sin \pi \varepsilon}+\frac{\pi^{2}}{3}\right\} .
\end{aligned}
$$

The calculation of (4.23) follows the same line but is more lengthy. We will not present it here but give only some comments. In this case the second power of the combination $\alpha u+1-u$ appears in the denominator at the stage (A4). It is useful then to perform integration by parts, reducing the power by one. The rest is similar to the calculations of (A1). The final answer is

$$
\begin{aligned}
& -\kappa^{4} z_{0} c_{0} \sum_{l>s} l \cdot \int_{p, q} D_{p}(s) D_{p}^{c}(s) D_{q}^{c}(l) D_{\mathbf{p}+\mathbf{q}}(s+l) \\
& \longrightarrow \Omega_{D}^{2} h_{0}^{4 \varepsilon} \cdot \frac{\mathrm{I}_{3}(\varepsilon)}{2 \varepsilon^{2}}\left\{-\frac{2}{\alpha} \cdot \frac{[\Gamma(1-\varepsilon)]^{2}}{\Gamma(1-2 \varepsilon)} \cdot \frac{(1+z)^{\varepsilon}-(1+\alpha z)^{\varepsilon}}{z(1-\alpha)(1+\varepsilon)}\right. \\
& \left.-\frac{\alpha^{-\varepsilon}-1}{\varepsilon} \cdot \frac{\pi \varepsilon}{\sin \pi \varepsilon}-2 \ln \alpha \cdot \frac{[\Gamma(1-\varepsilon)]^{2}}{\Gamma(1-2 \varepsilon)}\left[1+\frac{(1+z)^{\varepsilon}-1}{z}\right]\right\} .
\end{aligned}
$$

The last contribution (4.24) is finite and of no interest. 


\section{REFERENCES}

* Permanent address: Russian Research Center "Kurchatov Institute", Kurchatov sq. 1, 123182 Moscow, Russia.

$\dagger$ The phrase 'free energy' in this problem actually stands for the grand canonical potential. This terminology is used only to stress the analogy with $Q$-field theory for free electrons.

${ }^{1}$ A.M.M. Pruisken and M.A. Baranov, Europhys. Lett. 31, 543 (1995)

2 See, e.g., A.M.M. Pruisken and H.P. Wei, AIP Conf. Proc. 286, 159 (1994)

${ }^{3}$ H.P. Wei, D.C. Tsui and A.M.M. Pruisken, Phys. Rev. B33, 1488 (1986)

${ }^{4}$ H.P. Wei, D.C. Tsui, M.A. Palaanen and A.M.M. Pruisken, Phys. Rev. Lett. 61, 1294 (1988)

${ }^{5}$ A.M.M. Pruisken, M. Baranov and B. Škorić, preprint ITFA 97-47, cond-mat/9712322

${ }^{6}$ A.M.M. Pruisken, B. Škorić and M.A. Baranov, preprint ITFA 98-17, cond-mat/9807241

${ }^{7}$ X.G. Wen, Phys. Rev. B41, 12838 (1990); Int. J. Mod. Phys. B6, 1711 (1992)

${ }^{8}$ A.M. Finkelstein, JETP Lett. 37, 517 (1983); Soviet Phys. JETP 59, 212 (1984); Physica B197, 636 (1994)

${ }^{9}$ C. Castellani, G. Kotliar, P.A. Lee, Phys. Rev. Lett. 59, 323 (1987)

${ }^{10}$ A.L. Efros and B.I. Shklovskii, J. Phys. C: Solid State Phys. 8, L49 (1975)

${ }^{11}$ A.M.M. Pruisken, Z. Wang, Nucl. Phys. B322, 721 (1989)

12 E. Brézin, S. Hikami and J. Zinn-Justin, Nucl. Phys. B165, 528 (1980)

13 A.M.M. Pruisken, Phys. Rev. B31, 416 (1985)

${ }^{14}$ D. Belitz and T.R. Kirkpatrick, Rev. Mod. Phys. 166, 261 (1994) 